\title{
Effects of turbulator with round hole on the thermo-hydraulic performance of nanofluids in a triangle tube
}

Cong Qi ${ }^{\text {a, b, c, * }}$, Fan Fan ${ }^{\text {a, b }}$, Yuhang Pan ${ }^{\text {a, b }}$, Maoni Liu ${ }^{\text {a, b}}$, Yuying Yan ${ }^{\text {c }}$

a Jiangsu Province Engineering Laboratory of High Efficient Energy Storage Technology and Equipments, China University of Mining and Technology, Xuzhou 221116, China

${ }^{\mathrm{b}}$ School of Electrical and Power Engineering, China University of Mining and Technology, Xuzhou 221116, China

${ }^{\mathrm{c}}$ Fluids \& Thermal Engineering Research Group, Faculty of Engineering, University of Nottingham, Nottingham NG7 2RD, UK

Abstract: For investigating the thermal and hydraulic characteristics of water-based

$\mathrm{SiO}_{2}$ nanofluids in a triangular tube with different turbulators, an experimental system has been designed and verified in this paper. The effects of different round hole diameters $(d=3 \mathrm{~mm}, 4 \mathrm{~mm}, 5 \mathrm{~mm})$ and round hole pitch-rows $(l=5 \mathrm{~cm}, 10 \mathrm{~cm}, 15 \mathrm{~cm})$ of perforated turbulators on the thermo-hydraulic characteristics are researched. Meanwhile, the influences of Reynolds numbers $(R e=400-8000)$ and nanoparticles mass fractions (D-I water, $\omega=0.1 \%, 0.3 \%, 0.5 \%)$ are also studied. These experimental results show that, under the same circumstance, the nanofluids in the triangular tube with $\omega=0.5 \%$ have the largest positive influence on the heat transfer enhancement ratio which is up to $16.73 \%$. For a comprehensive study of the flow and heat transfer, thermal efficiency (comprehensive performance index) and exergy efficiency are adopted. It can be found that the larger the diameter and the smaller the pitch-row of the holes is, the greater the comprehensive evaluation index can be. In addition, all working conditions exhibit the superior exergy efficiency. The highest exergy efficiency can be got when $R e=6000$ and $\omega=0.5 \%$.

Key words: Nanofluids; Forced convection; Thermal efficiency; Exergy efficiency

*Correspondence author.

E-mail: qicong@cumt.edu.cn (C. Qi); fanfan@cumt.edu.cn (F. Fan); panyuhang@cumt.edu.cn (Y. Pan); liumaoni@cumt.edu.cn (M. Liu); yuying.yan@nottingham.ac.uk (Y. Yan) 
28 Nomenclature

$29 A$

$30 b_{\mathrm{i}}$

$31 c_{1}, c_{2}$

$32 c_{\mathrm{p}}$

33

$34 \quad c_{\mathrm{pb}}$

35

$36 c_{\mathrm{pp}}$

37

$38 C_{Q, P}$

39

40

41

$42 C_{Q, V}$

43

44

45

46

47

48

49

50

51

52

$53 d$

$54 d_{1}$

$55 d_{2}$

$\begin{array}{lll}56 & d_{3}\end{array}$

$57 f$

$58 h$

59

$60 k$

61

$62 k_{\mathrm{i}}$

$63 l$

$64 L$

$65 \mathrm{Nu}$

$66 p$

$67 P$

$68 \Delta p / \Delta L$

69

$70 Q$

$71 q_{\mathrm{m}}$

rate

$\mathrm{Pa} \cdot \mathrm{m}^{-1}$ cross-sectional area, $\mathrm{m}^{2}$

intercept of straight line

coefficient in equation

heat capacity of nanofluids, $76 T_{\text {out }}$ $\mathrm{J} \cdot \mathrm{kg}^{-1} \cdot \mathrm{K}^{-1}$

$77 T_{\text {in }}$ heat capacity of base fluid, $78 T_{\mathrm{f}}$ $\mathrm{J} \cdot \mathrm{kg}^{-1} \cdot \mathrm{K}^{-1}$

79

heat capacity of nanoparticles, $80 T_{\mathrm{w}}{ }^{*}$ $\mathrm{J} \cdot \mathrm{kg}^{-1} \cdot \mathrm{K}^{-1}$

81

the ratio of heat transfer rate $82 T_{\mathrm{w}}$

between enhanced and 83

reference surfaces under $84 u$

identical pumping power

85 Greek symbols

the ratio of heat transfer rate $86 \delta$

between enhanced and $87 \eta$

reference surfaces over the ratio 88

of friction factor between $89 \lambda$

enhanced and reference 90

surfaces under identical flow $91 \mu$

$92 \xi$

the ratio of heat transfer rate 93

between enhanced and $94 \rho$

reference surfaces under $95 \rho_{\mathrm{p}}$

identical pressure drop $\quad 96 \rho_{\mathrm{pb}}$

diameter of circular hole, $\mathrm{mm} \quad 97 \rho_{\mathrm{pp}}$

outer diameter of tube, $\mathrm{m} \quad 98 \varphi$

equivalent diameter of tube, $m 99 \omega$

100 Subscripts

inner diameter of tube, $\mathrm{m}$

frictional resistance coefficient 101

$\mathrm{m}_{1}, \mathrm{~m}_{2}$

convective heat transferto2 in

coefficient, $\mathrm{W} \cdot \mathrm{m}^{-2} \cdot \mathrm{K}^{-1}$

103 out

thermal conductivity

ofi04 0

nanofluids, $\mathrm{W} \cdot \mathrm{m}^{-1} \cdot \mathrm{K}^{-1}$

$105 \mathrm{e}$

slope of straight line

$106 \mathrm{nf}$

pitch-row, cm

$107 \mathrm{f}$

$108 \mathrm{p}$

$109 P$

$110 R e$

pressure, $\mathrm{Pa}$

111

pitch of corrugated tube, $\mathrm{m}$

pressure drop per unit length112 $V$

$113 \Delta p$

heat absorbed by nanofluids, J 114 w

mass flow rate, $\mathrm{kg} \cdot \mathrm{s}^{-1}$

115 outside-radius of tube, $\mathrm{m}$

inner-radius of tube, $\mathrm{m}$

Reynolds number

outlet temperature of tube, $\mathrm{K}$

inlet temperature of tube, $\mathrm{K}$

average temperature of nanofluids, $\mathrm{K}$

outside surface temperature of tube, $\mathrm{K}$

inside surface temperature of tube, $\mathrm{K}$

velocity of nanofluids, $\mathrm{m} \cdot \mathrm{s}^{-1}$

thickness of tube,m

relative heat transfer

enhancement ratios

thermal conductivity of tube,

$\mathrm{W} \cdot \mathrm{m}^{-1} \cdot \mathrm{K}^{-1}$

dynamic viscosity, $\mathrm{Pa} \cdot \mathrm{s}$

comprehensive performance

index

density of fluid, $\mathrm{kg} \cdot \mathrm{m}^{-3}$

density of nanofluids, $\mathrm{kg} \cdot \mathrm{m}^{-3}$

density of base fluid, $\mathrm{kg} \cdot \mathrm{m}^{-3}$

density of nanoparticle, $\mathrm{kg} \cdot \mathrm{m}^{-3}$

volume fraction, $\%$

mass fraction, $\%$

exponent in equation

import

outport

circular tube

enhanced tube

nanofluids

base fluid

nanoparticle

under the same pumping power

under the same Reynolds

number

under the same mass flow rate under the same pressure drop wall 


\section{Introduction}

With the development of technology in heat transfer equipment, conventional heat transfer medium and smooth tube cannot satisfy with the request of the increasing heat exchange amount. In order to achieve much higher heat exchange amount, there is a need to seek new fluid and enhanced tubes.

In recent years, nanofluids have been widely applied in the field of heat exchange due to their excellent thermal conductivity. For example, full-spectrum photo-thermal conversion $[1,2]$, enhanced solar thermal conversion [3], defects-assisted solar absorption [4], solar steam generation [5, 6], enhanced pool boiling heat transfer on porous surface [7] and superhydrophilic surface [8], CPU cooling $[9,10,11]$.

As we all know, convection heat transfer is mainly divided into natural convection and forced convection. For natural convection, a plenty of scholars have explored it. Shi et al. [12] explored the natural convection of nano- $\mathrm{Fe}_{3} \mathrm{O}_{4} @ \mathrm{CNT}$ fluids, analyzed the influence of different directions and strength of magnetic fields, and proposed a controllable heat transfer method. Guo et al. [13] used Lattice Boltzmann Method (LBM) to simulate the natural convection of nanofluids in an enclosed field, and these results showed that heat transfer characteristics are improved by the increase of $R a$ number. Pordanjani et al. [14] explored the nanofluids based on nature convection in the cavity with various magnetic fields. The consequence revealed that thermal properties of the material can increase with the increasing magnetic field angle gradually. Nojoomizadeh et al. [15] researched the $\mathrm{Fe}_{3} \mathrm{O}_{4}-\mathrm{H}_{2} \mathrm{O}$ nanofluids 
flowing through a two dimensional microchannel whose bottom half is filled with porous medium. These consequences revealed that the heat transfer characteristics increase with the rising Darcy number in the non-porous region but decrease in the porous region. Teimouri et al. [16] explored the numerical simulation of laminar mixed convection in horizontal eccentric annulus. The consequences showed that $\mathrm{Nu}$ numbers augment with the rising of downward eccentricity of inner cylinder. Sheremet et al. explored the natural convection based on a cavity which is full of nanofluids. In addition, the effects of the inclination angle [17], Brownian diffusion and thermophoresis [18] were analyzed. These consequences revealed that the rate of heat exchange can increase as the growth of inclination angle, and Nusselt numbers show a decreasing function of the heater size. Miroshnichenko et al. studied the natural convection of open oblique cavities with heating elements [19] and open cavities with multiple porous layers [20] which were full of water-based $\mathrm{Al}_{2} \mathrm{O}_{3}$ nanofluids respectively. These above-mentioned results showed that the average Nusselt number with an inclination angle of $\pi / 3$ can show the largest value, and it increases with the volume fraction at $R a=10^{5}$ when the distance between the first porous layer and the left vertical wall is smaller than $\delta<0.1$. Pourmehran et al. explored the effects of external magnetic field [21, 22], rotational Reynolds number [23] and nanoparticle size [24] on nanofluids. These studies indicated that the large magnetic field, rotational Reynolds number and small nanoparticle size are helpful to reinforce the heat transfer. Izadi et al. studied the free convection of nanofluids in a $\perp$ shaped cavity [25], a porous undulant-wall enclosure [26], a porous enclosure under 
magnetic fields [27], and between two eccentric cylinders filled with porous material [28]. Results indicated that larger heat source aspect ratio, smaller Lewis number and higher magnetic number are beneficial to the heat transfer enhancement. Mahian et al. [29] used theoretical correlation to explore the heat transfer capability of silica nanofluids in square and triangle enclosures, and compared the calculated results with experimental data.

Researchers all over the world have also investigated the forced convection heat transfer. Shahsavani et al. [30] explored the thermo-hydraulic performance of non-Newtonian nanofluids in circular tubes and developed their new correlations to calculate the power law exponent, viscosity exponent and thermal conductivity. Sheikholeslami et al. explored the forced convection under a magnetic field, and discussed various influencing factors of heat transfer, which include cubic cavity driven by a porous cap [31], Lorentz force [32], Kleinstreuer-Li (KKL) model [33], shape of nanoparticles [34], electric field dependent viscosity [35], porous media under electric field [36], compound turbulator [37], and hot sphere obstacle [38]. Naphon et al. explored the convection heat transfer in helically corrugated tubes based on $\mathrm{TiO}_{2}$-water nanofluids [39] and a coil pipe [40, 41, 42] under magnetic field, and analyzed the effects of pulsating flow frequency [39], magnetic displacement [40], and magnetic orientation [41] on the convection heat exchange. Also, the forced convective heat transfer with pulsating nanofluids was explored by applying artificial neural networks [42]. The above results indicated that additions of pulsating flow and magnetic field are advantageous to intensify the heat transfer. Zhou et al. [43] 
explored the numerical simulation on the forced convection heat transfer of nanoparticle-metal fluid in circular tubes. The impacts of $R e$ and volume percentage of nanoparticle were discussed. It was found that the nanoparticle-metal fluid shows higher heat exchange performance than that of nanoparticle-water. Sun et al. have finished a series of experiments on the forced convection heat transfer in external thread tube [44] and internal thread tube [45] with nanometer refrigerant. The impacts of various nanofluids as well as nanoparticle mass fractions on the thermo-hydraulic characteristics were explored, and the conclusion showed that water-based $\mathrm{Cu}$ nanofluids demonstrated an excellent heat transfer characteristic compared with water-based $\mathrm{Al}$, water-based $\mathrm{Al}_{2} \mathrm{O}_{3}$, and water-based $\mathrm{Fe}_{2} \mathrm{O}_{3}$ nanofluids. Also, it was found that enhanced heat transfer effect in the internal thread tube shows a larger improvement. Qi et al. experimentally explored the compulsive convection heat transfer based on nanofluids flow through triangular tubes with turbulator [46, 47], corrugated tubes under magnetic induction intensity [48] and circular tubes under magnetic induction intensity [49], circular tubes with rotating turbulators [50], horizontal elliptical tubes [51], and corrugated tubes [52]. Mohebbi et al. [53] simulated the forced convection of nanofluids in an extended surfaces channel by lattice Boltzmann method. It could be found that these tubes and enhanced technologies tend to reinforce the heat transfer. Minakov et al. [54] studied the forced convection of silica nanofluids in cylindrical channels and found that the heat transfer capacity is affected by factors such as nanoparticle size and fluid inlet temperature. Based on the circular tube with the turbulator, Sundar et al. [55] carried out an 
experimental study on the thermal hydraulic characteristics of alumina nanofluid. It was found that the $N u$ number of the nanofluids is increased by $33.51 \%$ and the drag coefficient is increased by $9.6 \%$ compared with the deionized water. Man et al. [56] compared the alternation of clockwise and counterclockwise turbulator and typical turbulator, and studied their enhancement of heat transfer. It was found that the former has better heat transfer performance.

The above experts and scholars have explored the enhancement of heat transfer of nanofluids with different cavities and different working conditions in detail, and have obtained abundant research results. It can be noticed that researches on intensified heat transfer tubes are mainly based on round tubes, fluted tubes and corrugated tubes, but rarely based on triangular tubes. Therefore, the purpose of this paper is to investigate the effects of round hole diameter and pitch-row on thermal and hydrodynamic characteristics of nanofluids based on triangular tubes with perforated turbulator inserted, and apply thermal and exergy efficiency to assess the comprehensive thermal and hydrodynamic characteristics. The innovation of this paper lies in two following respects. Firstly, triangular tubes and turbulators are combined to analyze the thermal and hydraulic characteristics of nanofluids. Secondly, the working conditions are comprehensively evaluated from thermal efficiency and exergy efficiency.

\section{Experimental method}

\subsection{Experimental system}

Based on the previous analyses, this paper intends to adopt $\mathrm{SiO}_{2}-\mathrm{H}_{2} \mathrm{O}$ nanofluids 
as the heat transfer medium. In the experimental system of this paper, the dispersant and particle acquisition of nanofluids are independent of each other, so the two-step method is more suitable. Here the particles selected in this paper are TSP-H10 $\mathrm{SiO}_{2}$ nanoparticles. The particles have a primary particle size of only $20 \mathrm{~nm}$, which makes it have a strong Brownian motion in the base fluid and reduce the sedimentation caused by gravity. Meanwhile, the particles have a large specific surface area, and it means that the particles have a strong surface activity, which makes the particles prone to agglomeration. Considering above reasons, in the process of preparing the working fluids, some measures are needed to maintain the stability of the nanofluids. At present, the commonly used methods are "Static stabilization" [57]. The method adjusts the $\mathrm{pH}$ of the nanofluids by adding an electrolyte solution such as $\mathrm{NaOH}$, so that the TSP-H10 $\mathrm{SiO}_{2}$ nanoparticles have the same kind of electric charge and mutually repel, thereby reducing agglomeration between particles. Another method is "Steric hindrance stabilization". The method is to add surfactants into the nanofluids, and the surfactant molecules cover the surface of TSP-H10 $\mathrm{SiO}_{2}$ nanoparticles to form a film to increase the repulsive force between the particles, thereby preventing the development and growth of the particle agglomerates. The last method is "Physical dispersion". Ultrasonic vibration, mechanical agitation and magnetic stirring are used to destroy the agglomeration of nanoparticles and achieve the effect of dispersing particles.

Fig. 1 represents a two-step process for preparing $\mathrm{SiO}_{2}$-water nanofluids. Firstly, the dispersant (TDL-ND1, $\omega=6 \%$ ) is added into the base solution, and it needs to take 
at least 30 minutes to stir the liquids mechanically. Secondly, add nanoparticles $(\omega=0.1 \%, 0.3 \%, 0.5 \%)$ and stir the mixture for an hour at least. Then, the $\mathrm{pH}$ is adjusted to 8 with a sodium hydroxide solution, and the liquids still need to be stirred for 1 hour. Finally, use ultrasonic wave to oscillate the fluids $40 \mathrm{~min}$. After above steps, stable $\mathrm{SiO}_{2}-\mathrm{H}_{2} \mathrm{O}$ nanofluids with various nanoparticle mass fractions are prepared successfully (see Fig. 2).
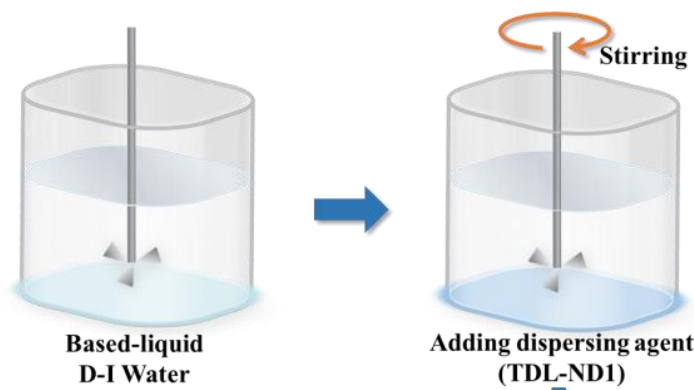

Adding dispersing agent

D-I Water
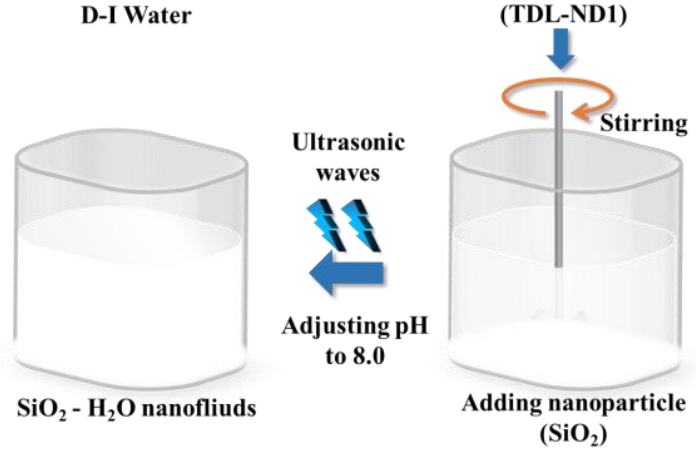

Fig. 1. Procedure of producing $\mathrm{SiO}_{2}$-water nanofluids by a two-step method
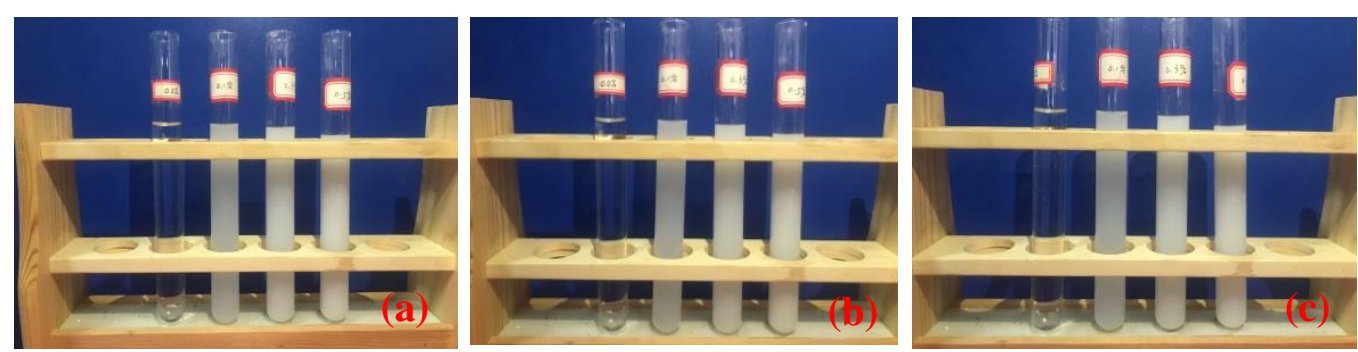

Fig. 2. $\mathrm{SiO}_{2}$-water nanofluids at different quiescent time, (a) just prepared, (b) laying up 10 days, (c) laying up 20 days

After the preparation of stable nanofluids, the stability of the nanofluids is

analyzed, including mechanical agitation and ultrasonic oscillation time, dispersant 
mechanical agitation time is at least 150 minutes, in order to fully mix the base liquid, dispersant and nanoparticles. Meanwhile, the ultrasonic oscillation time is preferably 40 minutes. If the time is too short, the particle dispersion will not be sufficient. If the time is too long, the fluid temperature will rise and the stability of the nanofluid will be affected. Fig. 3 shows the change of transmittance with the quiescent time of nanofluids under different dispersant concentrations and different $\mathrm{pH}$ values. It can be seen that the $\mathrm{SiO}_{2}-\mathrm{H}_{2} \mathrm{O}$ nanofluids exhibit the best stability (lowest transmittance) when $\mathrm{pH}=8$ and the dispersant concentration $m=6 \mathrm{wt} \%$. prepared working fluids meet the stability requirements of the experiment.
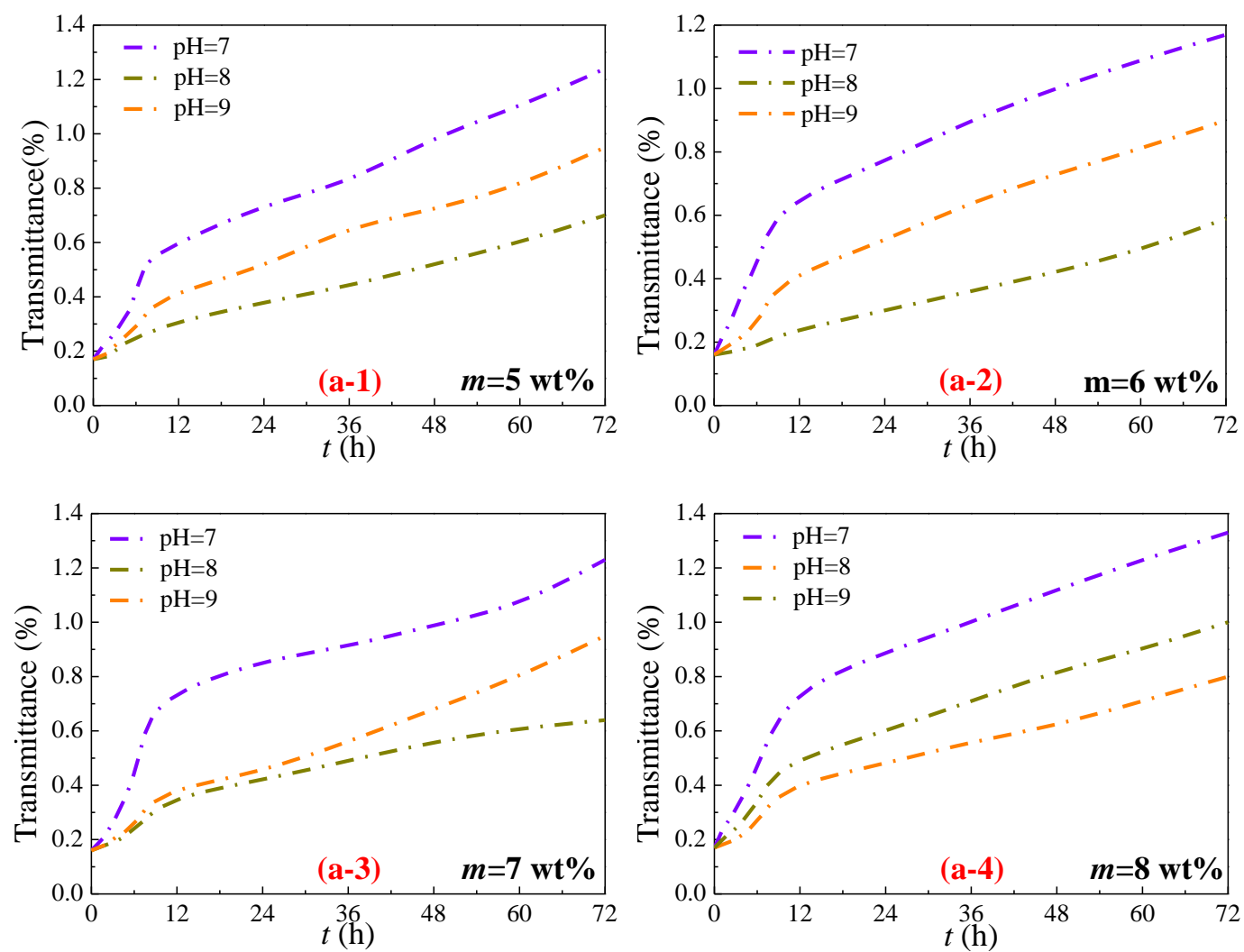

273 

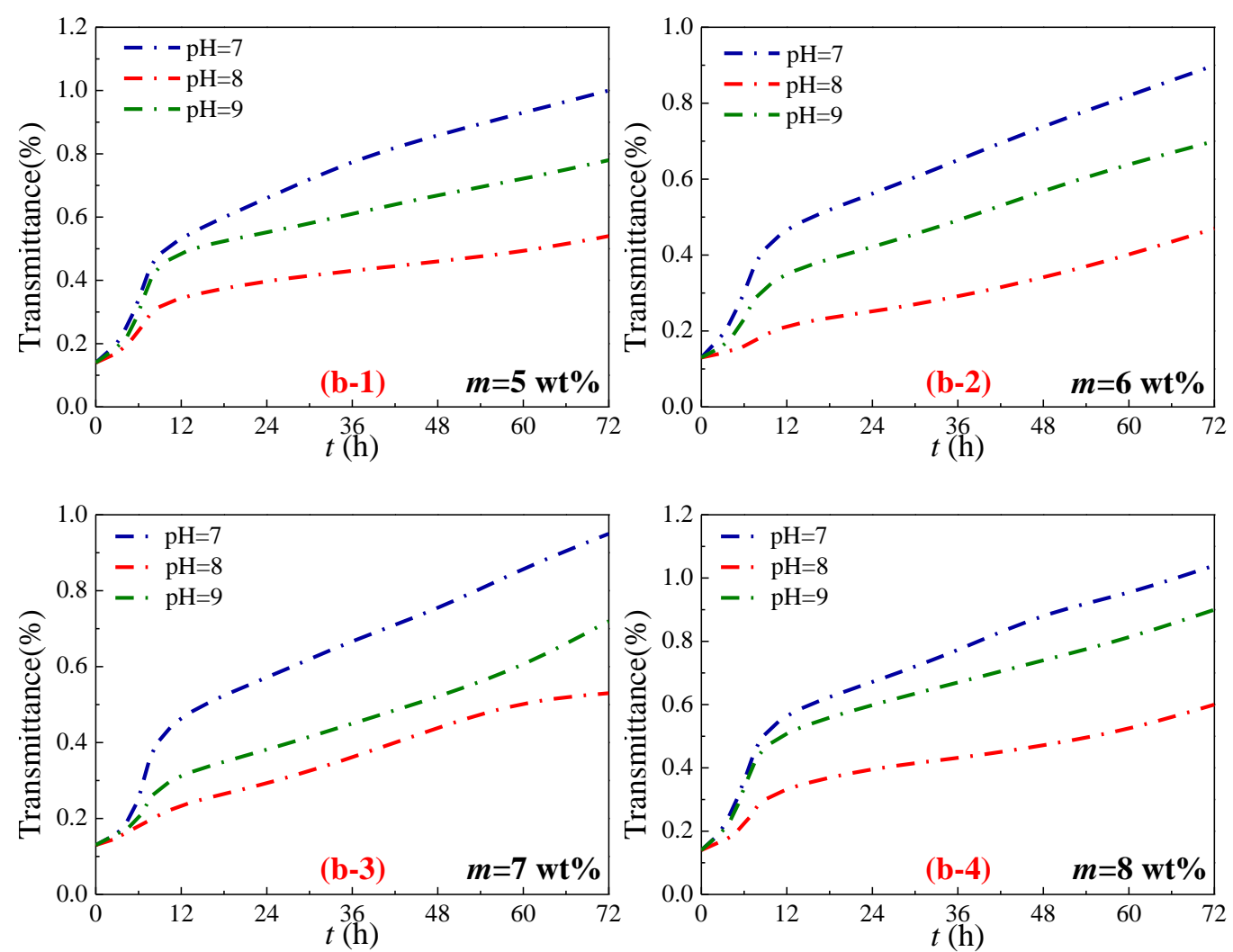

275
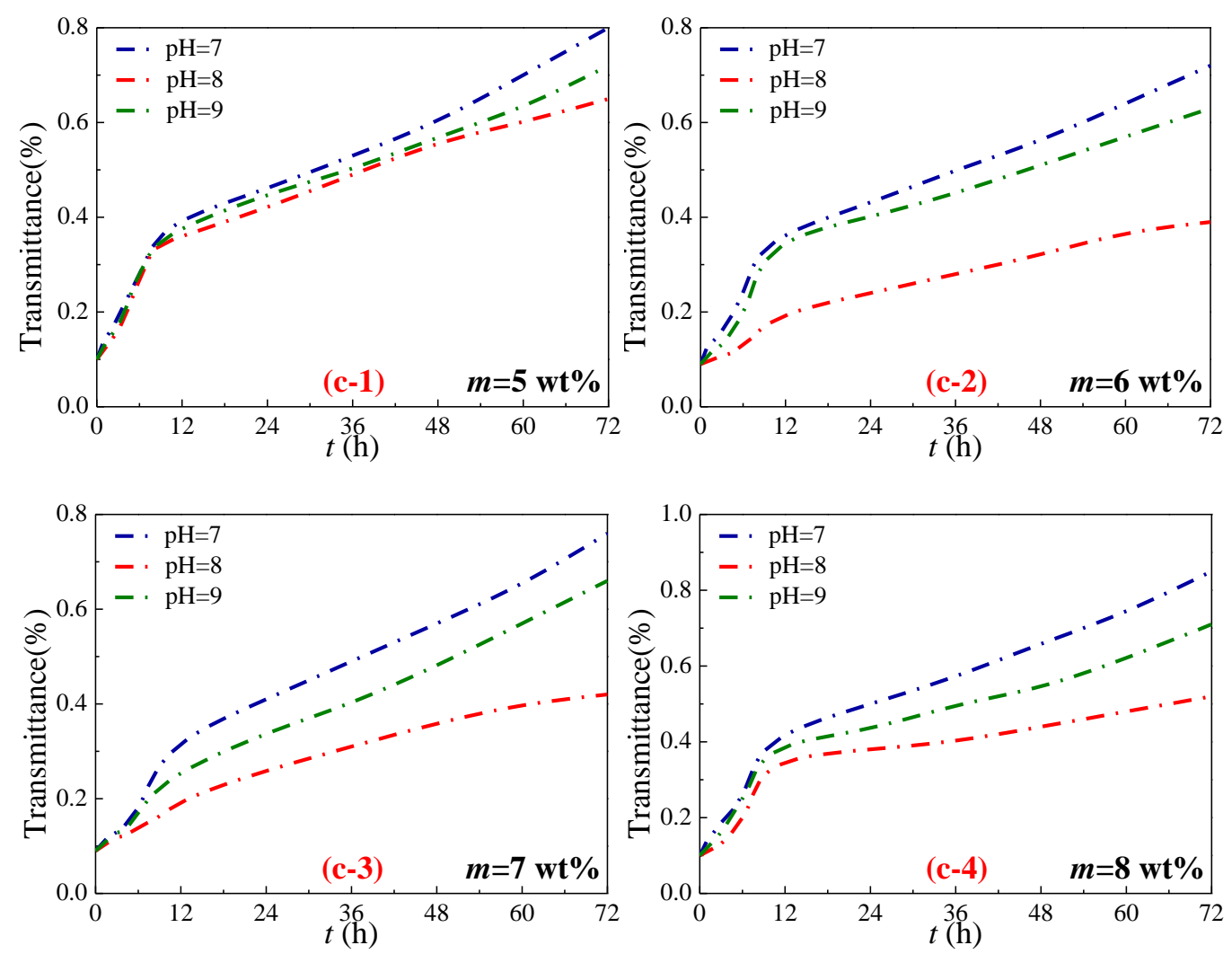

Fig. 3 Transmittance changes with quiescent time of $\mathrm{SiO}_{2}$-water nanofluids under different dispersant concentrations and $\mathrm{pH}$ values, (a) $\omega=0.1 \%$, (b) $\omega=0.3 \%$, (c) $\omega=0.5 \%$

After preparing the nanofluids with stable properties, the thermophysical 
commonly used experimental correlations. They are shown below (see Fig. 4).

284

285
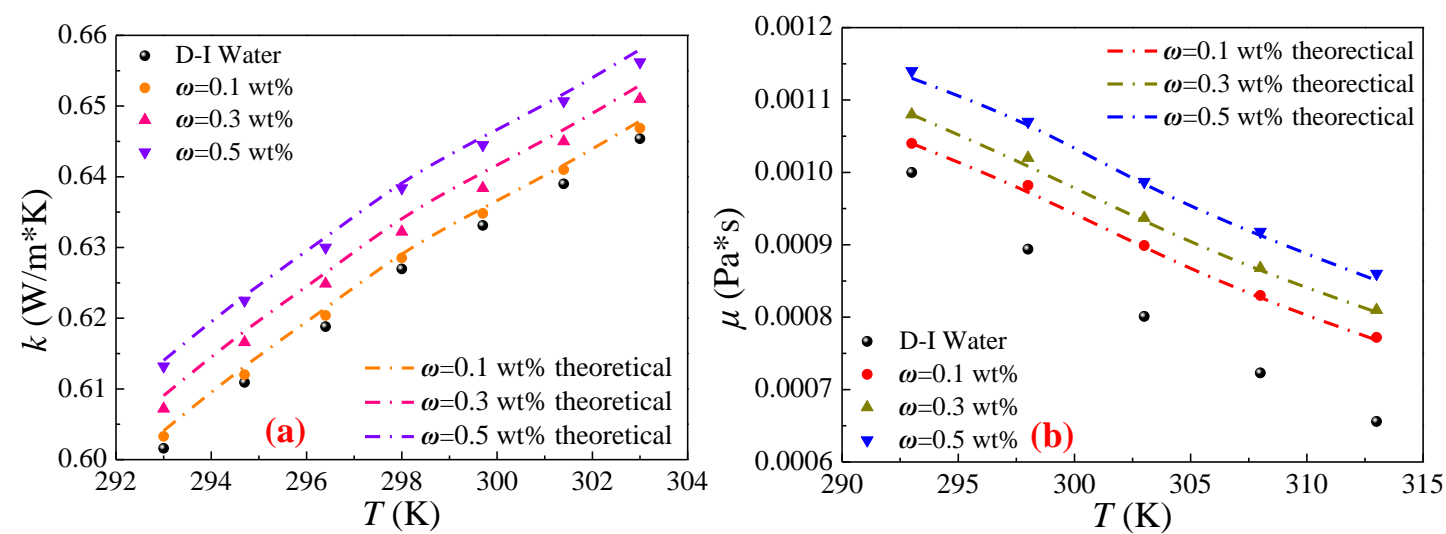

Fig. 4 Comparison of experimental and theoretical values of thermophysical properties of $\mathrm{SiO}_{2}-\mathrm{H}_{2} \mathrm{O}$ nanofluids, (a) thermal conductivity, (b) viscosity

Through continuous testing and analysis of experimental data, Maxwell [58] has

obtained an empirical formula for calculating the thermal conductivity of nanofluids:

$$
\frac{k_{n f}}{k_{f}}=\frac{k_{p}+2 k_{f}-2 \varphi\left(k_{f}-k_{p}\right)}{k_{p}+2 k_{f}+\varphi\left(k_{f}-k_{p}\right)}
$$

Through the analysis of experimental data, an empirical formula for calculating

the viscosity of nanofluids is obtained [59]:

$$
\mu_{n f}=\frac{\mu_{f}}{(1-\varphi)^{2.5}}
$$

The schematic diagram of the experimental system is exhibited in Fig. 5. From this, it can be seen that the test part is made up of two parts, one is the heat transfer test section, and the other is the resistance test section. The former is the primary part in the experimental system, and it consists of triangular tube and perforated turbulators. The round hole is chosen because the area of the circle is the largest when the perimeter is the same. Other shapes of holes (such as triangular holes, rectangular holes) is our follow-up work. The turbulator is placed in the middle of the triangular 
tube and the length is consistent with the length of the tube. The structures of

301

302

303

304

305

306

307

308

309

310

311

312

313

triangular tube and turbulator are shown in Fig. 6. Resistor wires are wound around the triangular tube to heat it, which are connected to a DC power. The wall temperature of the triangular tube is measured by nine T-type thermocouples. In the meantime, at both ends of the tube, a pair of armored thermocouples (accuracy: $\pm 0.1 \%$ ) is arranged along the flow direction of the experimental system. The temperature collected by thermocouple is gathered by Agilent data acquisition system.

Considering the loss of heat dissipation, the triangular tube is wrapped in insulating cotton. To analyze the heat exchange characteristics of $\mathrm{SiO}_{2}$ nanofluids from the flow view, the pressure difference transmitter is used to calculate the flow resistance. The cooling of the experimental system is implemented by a cryostat tank.
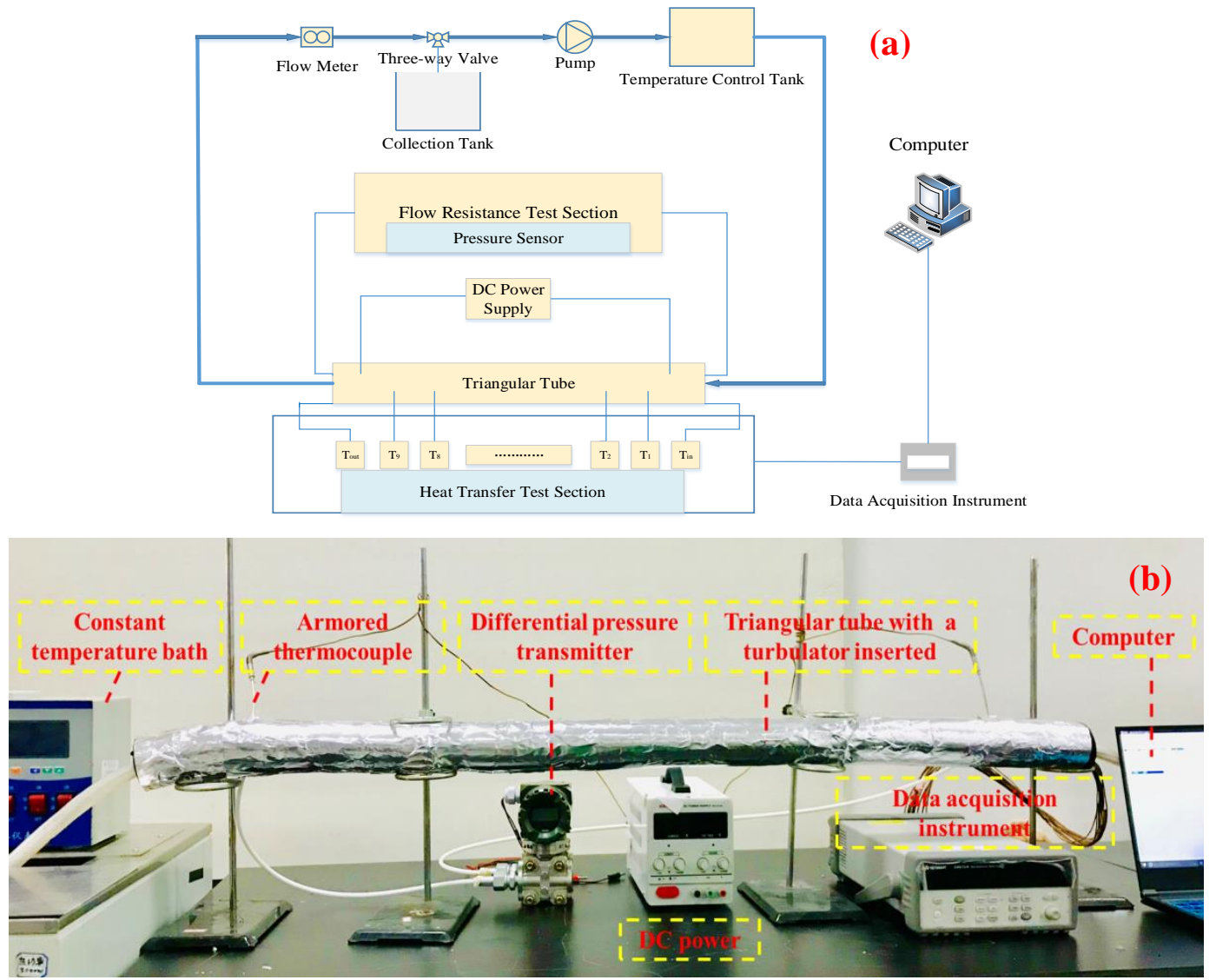

Fig. 5. The experimental system: (a) Schematic diagram, (b) Physical diagram 


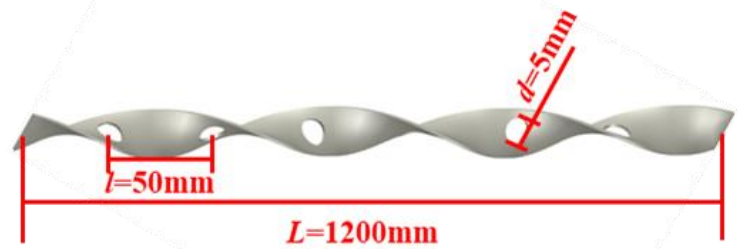

(b)

Fig. 6. The structure of triangular tube and turbulator with round holes, (a) triangular tube, (b) turbulator with round holes (take $d=5 \mathrm{~mm}, l=50 \mathrm{~mm}$ as example)

\subsection{Experimental data processing}

The equivalent diameter of the triangular tube can be obtained by the following formula:

$$
d_{\mathrm{e}}=\frac{4 A_{\mathrm{c}}}{P^{\prime}}
$$

And two equations [60] of specific heat and density of nanofluids are given:

$$
c_{\mathrm{p}}=(1-\varphi) c_{\mathrm{pf}}+\varphi c_{\mathrm{pp}}
$$

$$
\rho=(1-\varphi) \rho_{\mathrm{f}}+\varphi \rho_{\mathrm{p}}
$$

The heat absorbed by the fluids can be calculated as follows:

$$
Q_{\mathrm{f}}=c_{\mathrm{p}} q_{\mathrm{m}}\left(T_{\mathrm{out}}-T_{\mathrm{in}}\right)
$$

The following formula is used to calculate the nanofluids temperature flowing through the tube:

$$
T_{\mathrm{f}}=\frac{T_{\text {in }}+T_{\text {out }}}{2}
$$

And the following formula is used to calculate the exterior surface average temperature:

$$
T_{\mathrm{wo}}=\left[\sum_{i=1}^{9} T_{\mathrm{wo}}(i)\right] / 9
$$

Subsequently, the interior surface formula is obtained: 


$$
T_{\mathrm{wi}}=T_{w o}-\frac{Q_{f} \ln \left(r_{o} / r_{i}\right)}{2 \pi \lambda l}
$$

335 where $r_{\mathrm{i}}=\frac{d_{\mathrm{e}}}{4}, r_{\mathrm{o}}=r_{\mathrm{i}}+\delta_{\text {tube }}$.

Reynolds number is computed as:

337

338

340

341

$$
C_{Q, i}=\left(\frac{N u_{\mathrm{e}}}{N u_{0}}\right)_{R e} /\left(\frac{f_{\mathrm{e}}}{f_{0}}\right)_{R e}^{k_{i}}(i=P, \Delta p, V)
$$

$$
R e=\frac{\rho u d_{\mathrm{e}}}{\mu_{\mathrm{f}}}
$$
coefficient:

$$
h=\frac{Q_{\mathrm{f}}}{\pi d_{\mathrm{e}} l\left(T_{w i}-T_{\mathrm{f}}\right)}
$$
resistance coefficient [44]:

$$
N u=\frac{h d_{\mathrm{e}}}{\lambda_{\mathrm{f}}}
$$

$$
f=\frac{2 d \mathrm{e}}{\rho u^{2}} \cdot \frac{\Delta p}{\Delta l}
$$
method-comprehensive evaluation index [44] is put forward:

$$
\eta=\left(\frac{N u_{\mathrm{nf}}}{N u_{\mathrm{bf}}}\right) /\left(\frac{f_{\mathrm{nf}}}{f_{\text {bf }}}\right)^{\frac{1}{3}}
$$
nanofluids. The exergy efficiency is calculated as follows [61]:

The following method is used to calculate the convective heat exchange

Lastly, two following formulas are adopted to calculate the Nusselt number and

Adding nanoparticles can augment the whole thermal conductivity and increase the viscosity of fluids. To explore the quantity of energy, a

Considering the quality of energy in the heat transfer in these enhanced tubes, exergy efficiency criterion is introduced to assess the energy quality based on

where $f_{0}(R e)=c_{1} R e^{\mathrm{m}_{1}}, \quad N u_{0}(R e)=c_{2} R e^{\mathrm{m}_{2}}, \quad k_{P}=\frac{\mathrm{m}_{2}}{3+\mathrm{m}_{1}}, \quad k_{\Delta p}=\frac{\mathrm{m}_{2}}{2+\mathrm{m}_{1}}, \quad k_{V}=1$. 
And $P$ refers to identical pump work, $\Delta p$ refers to the same pressure loss, and $V$ refers to identical flow velocity.

Take the logarithm of both sides of formula (15):

$$
\ln \left(\frac{N u_{\mathrm{e}}}{N u_{0}}\right)_{R e}=b_{i}+k_{i} \ln \left(\frac{f_{\mathrm{e}}}{f_{0}}\right)_{R e}
$$

where $b_{P}=\ln C_{Q, P}, \quad b_{\Delta p}=\ln C_{Q, \Delta p}, \quad b_{V}=\ln C_{Q, V}, \quad-1 \leq \mathrm{m}_{1}<0, \quad 0<\mathrm{m}_{2}<1$.

\subsection{Uncertainty analysis}

To maintain the accuracies at a high level, uncertainty analysis based on the experimental system is needed. The uncertainty formulas for resistance coefficient and Nusselt number are as follows [62]:

$$
\begin{gathered}
\frac{\delta f}{f}=\sqrt{\left(\frac{\delta p}{p}\right)^{2}+\left(\frac{\delta l}{l}\right)^{2}+\left(\frac{\delta q_{\mathrm{m}}}{q_{\mathrm{m}}}\right)^{2}} \\
\frac{\delta N u}{N u}=\sqrt{\left(\frac{\delta Q_{\mathrm{f}}}{Q_{\mathrm{f}}}\right)^{2}+\left(\frac{\delta T}{T}\right)^{2}}
\end{gathered}
$$

Table 1 exhibits the accuracy of the variables in the experiment. By substituting above formulas, the uncertainty of drag coefficient and $\mathrm{Nu}$ number can be obtained to be $\pm 5.0 \%$ and $\pm 1.18 \%$ respectively, which means that the system has a good reliability.

Table 1 Accuracies of variables in the experiment

\begin{tabular}{cccccc}
\hline Variables & $Q_{\mathrm{f}}$ & $T$ & $p$ & $q_{\mathrm{nf}}$ & $l$ \\
\hline Uncertainties & $\pm 5.0 \%$ & $\pm 0.1 \%$ & $\pm 0.5 \%$ & $\pm 1.06 \%$ & $\pm 0.1 \%$ \\
\hline
\end{tabular}

\section{Results and discussions}

\subsection{Experimental system validation}

Apart from the uncertainty analysis, the experimental system is needed to be validated. From Fig. 7, it can be seen the comparison between the theoretical and 
experimental values of $N u$ and $f[60,63,64,65]$. According to the results of the

375 comparison, it can be found that the error between the theoretical and the experimental value is tiny, no more than $4 \%$, which indicates that the reliability of the experimental setup can be guaranteed.
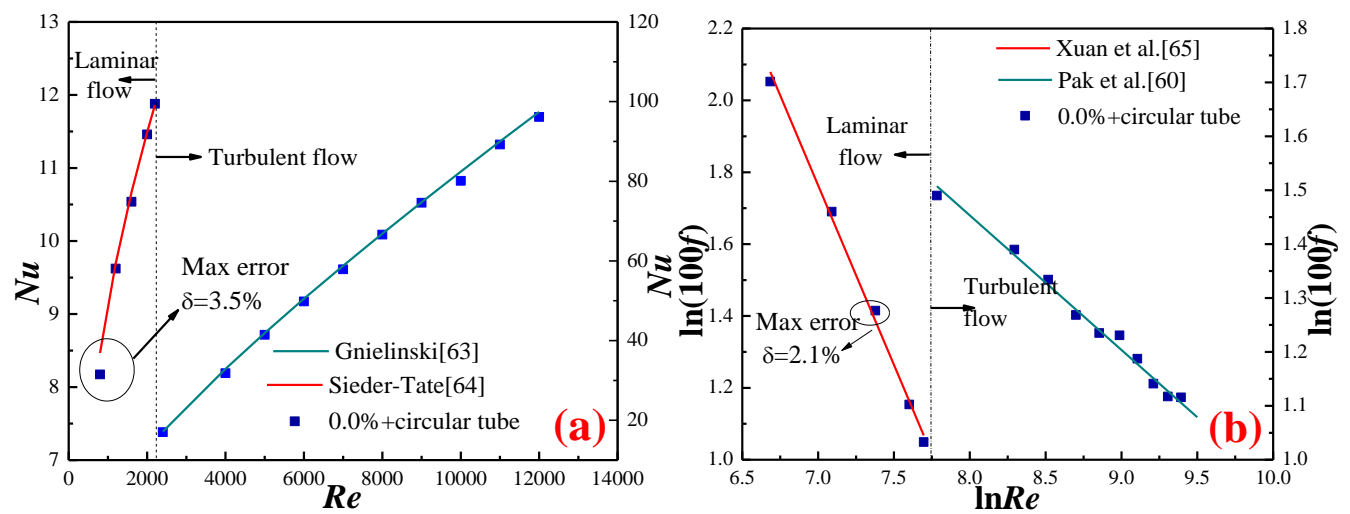

Fig. 7. Experimental verification, (a) $N u$, (b) $f$

\subsection{Experimental Results and discussions}

\subsubsection{Nusselt number}

First of all, it is necessary to explore the effect of turbulator on the heat transfer of $\mathrm{SiO}_{2}-\mathrm{H}_{2} \mathrm{O}$ nanofluids in a triangular tube. Fig. 8 reflects the convective heat transfer capability of working fluids in the triangular tube with or without turbulator. It is easy to find the following conclusions: Three mass fraction nanofluids can augment the heat transfer effect by $11.745 \%, 13.607 \%$ and $16.252 \%$ respectively compared with deionized water. The heat transfer effects of working fluids with a turbulator in the triangular tube are $11.26 \%, 11.97 \%$ and $14.32 \%$ higher than that of without turbulator. The heat transfer enhancement mechanism of turbulator mainly includes the following two aspects. For one thing, after the turbulator is inserted into the tube, the flow in it changes from the original horizontal flow to a three-dimensional rotating flow. The rotating flow increases the flow path and 
improves the turbulence intensity at the tube wall, which intensifies the disturbance of the boundary layer. This disturbance promotes the mixing of the boundary layer fluid with the mainstream, weakens the flow boundary layer, and effectively strengthens the convective heat transfer. For another, the secondary flow generated by turbulator is also one of the main reasons for the enhancement of heat transfer. As early as 1964, Smithberg et al. [66] had proposed that secondary flow can occur when the fluid was rotating. Due to the existence of the rotating flow, $\mathrm{SiO}_{2}-\mathrm{H}_{2} \mathrm{O}$ nanofluids are also subjected to centrifugal force. However, because of the viscous force of the wall, the tangential velocity of the fluid near the wall is lower than that farther away from the wall, which causes the fluid particles farther away from the wall to do centrifugal motion, while the fluid particles closer to the wall are subjected to less centrifugal force and do centripetal motion. The relative motion of the two types fluid particles leads to the generation of secondary flow and increases the flow turbulence, which enhances the heat transfer capacity.

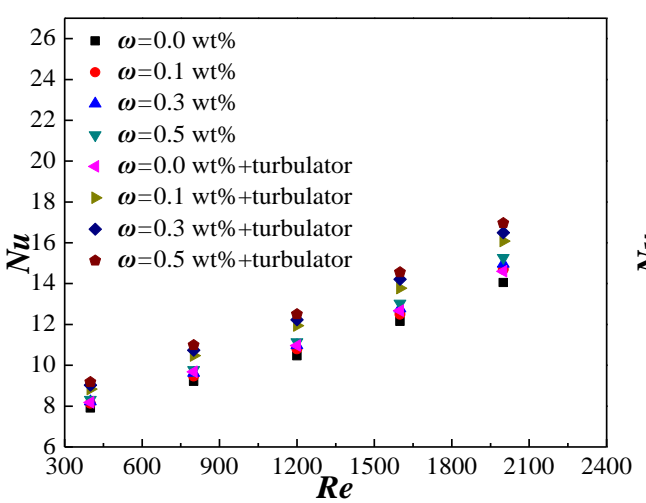

(a)

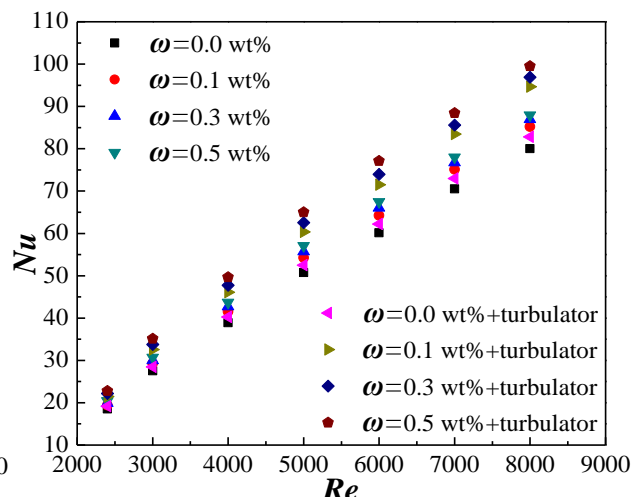

(b)

Fig. 8 The change of $\mathrm{Nu}$ numbers of $\mathrm{SiO}_{2}-\mathrm{H}_{2} \mathrm{O}$ nanofluids in the triangular tube with/without a turbulator: (a) laminar flow, (b) turbulent flow

Effects of mass fraction of nanoparticles on Nusselt number of triangular tubes 

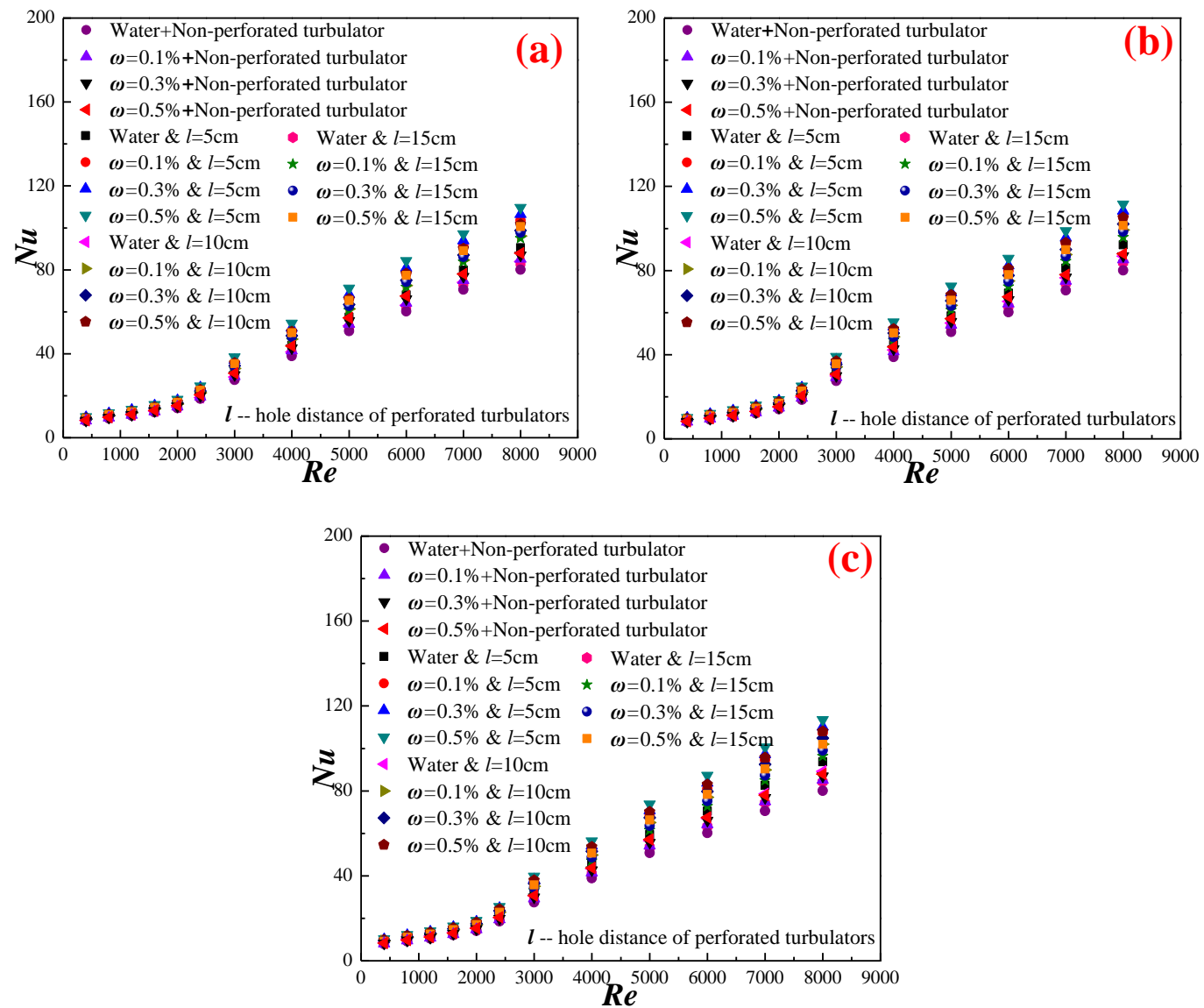

Fig. 9. Changes of Nusselt numbers with Reynolds numbers, (a) $d=3 \mathrm{~mm}$, (b) $d=4 \mathrm{~mm}$, 


\subsubsection{Effect of nanoparticle mass fraction}

Effects of nanoparticle mass fraction in the triangular tube with internally inserted turbulators on the heat transfer of working fluid are studied. For triangular tube with a perforated turbulator that diameter $d=3 \mathrm{~mm}$ in Fig. 10, the effect of heat transfer is the best obviously when the mass fraction is $0.5 \%$. Nanofluids with $\omega=0.5 \%$ can enhance the heat transfer by $16.51 \%, 16.49 \%, 16.48 \%$ in different round hole pitch-rows $(l=5 \mathrm{~cm}, l=10 \mathrm{~cm}, l=15 \mathrm{~cm})$ at most compared with D-I water respectively. For triangular tube with a perforated turbulator that diameter $d=4 \mathrm{~mm}$ in Fig. 11, nanofluids with $\omega=0.5 \%$ can enhance the heat transfer by $16.63 \%, 16.62 \%, 16.59 \%$ in different round hole pitch-rows $(l=5 \mathrm{~cm}, l=10 \mathrm{~cm}, l=15 \mathrm{~cm})$ at most
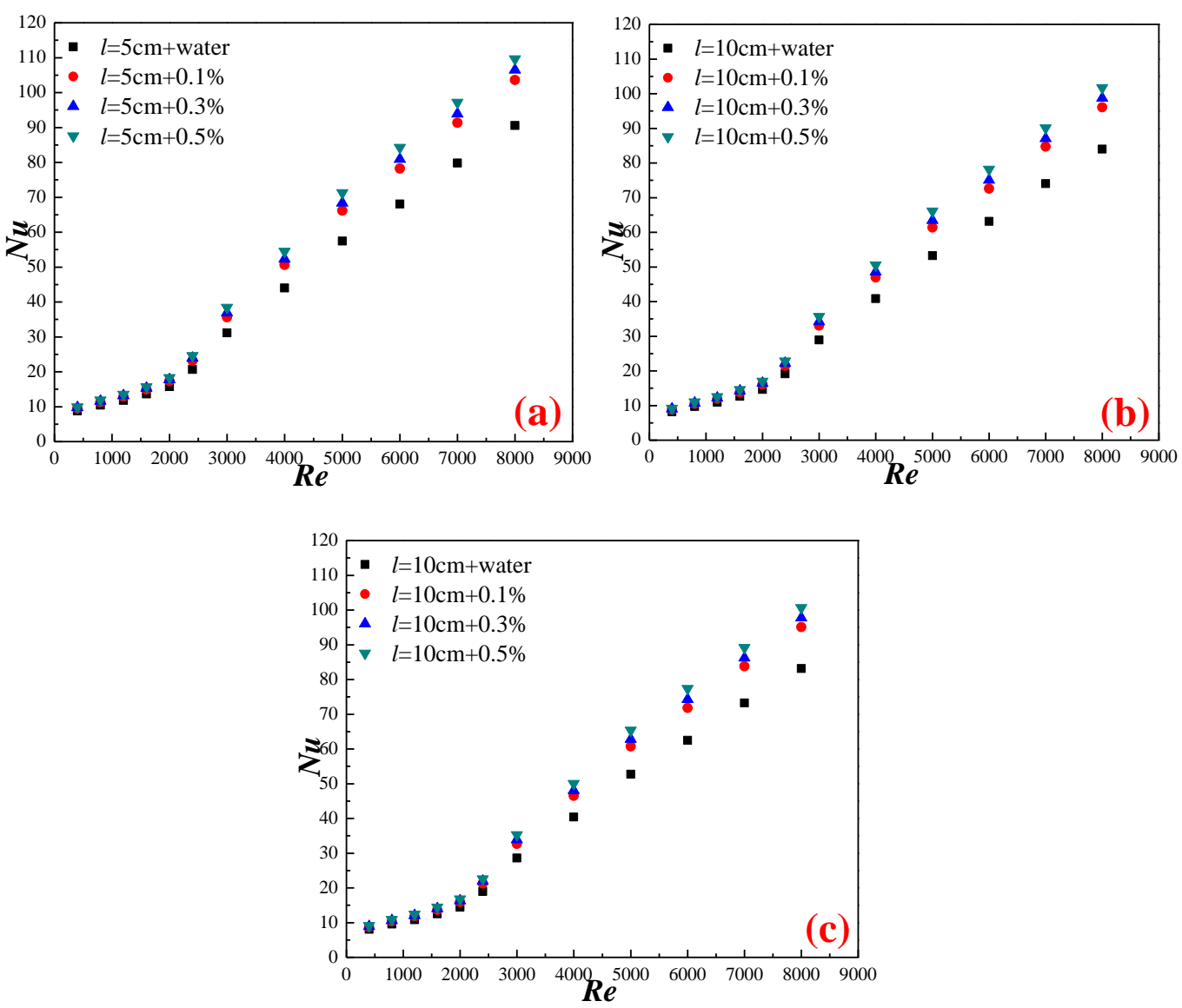

Fig. 10. Effects of nanoparticle mass fractions on Nusselt numbers of triangular tube, $d=3 \mathrm{~mm}$ : (a) $l=5 \mathrm{~cm}$, (b) $l=10 \mathrm{~cm}$, (c) $l=15 \mathrm{~cm}$ 

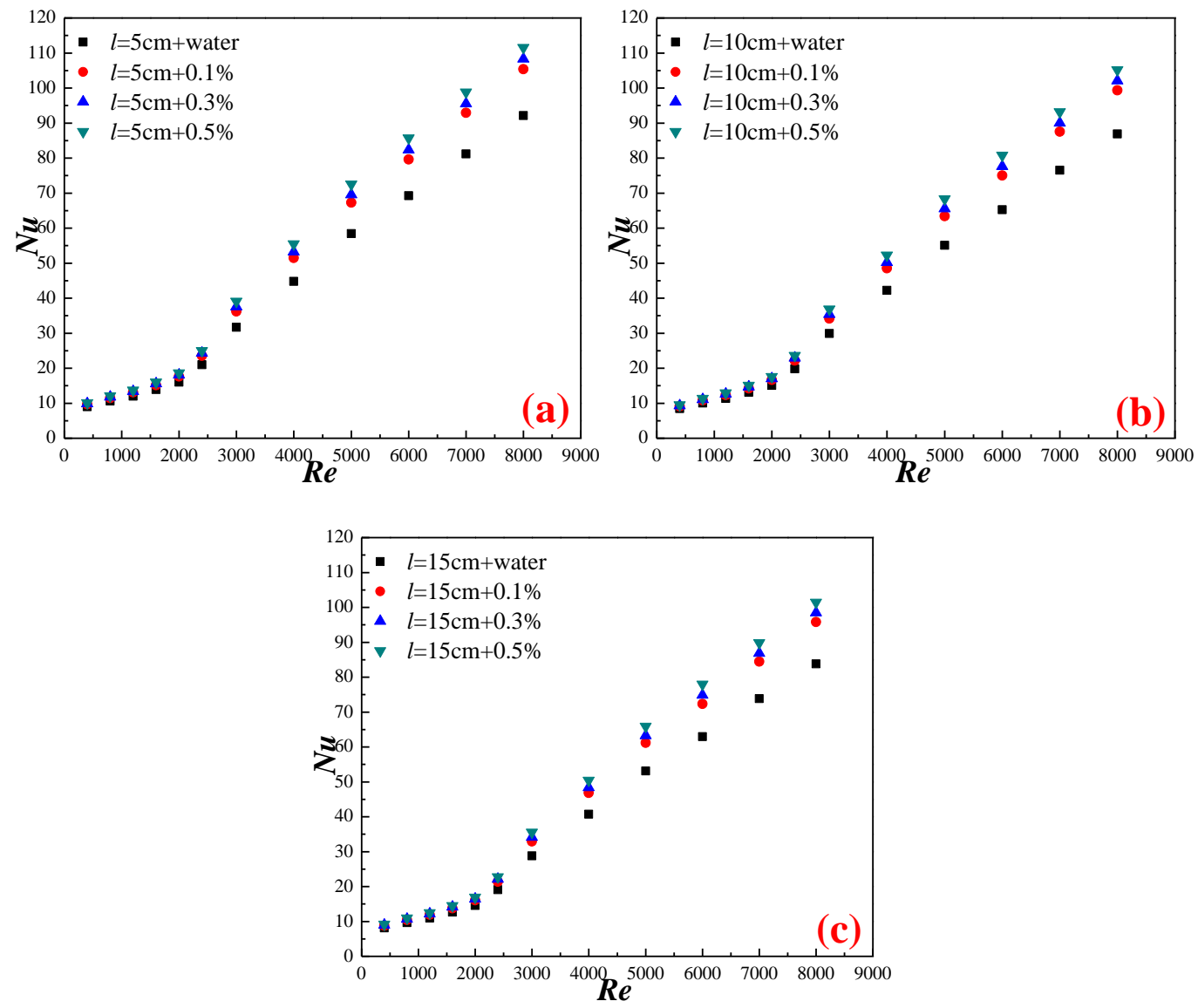

Fig. 11. Effects of nanoparticle mass fractions on Nusselt numbers of triangular tube,

$$
d=4 \mathrm{~mm} \text { : (a) } l=5 \mathrm{~cm} \text {, (b) } l=10 \mathrm{~cm} \text {, (c) } l=15 \mathrm{~cm}
$$

compared with D-I water respectively. For triangular tube with a perforated turbulator that diameter $d=5 \mathrm{~mm}$ in Fig. 12, nanofluids with $\omega=0.5 \%$ can enhance the heat transfer by $16.73 \%, 16.71 \%, 16.66 \%$ in different round hole pitch-rows $(l=5 \mathrm{~cm}$, $l=10 \mathrm{~cm}, l=15 \mathrm{~cm})$ at most compared with D-I water.

In a word, under different conditions, the maximum heat transfer efficiency occurs when the mass fraction reaches the highest, which can enhance the heat transfer by $16.73 \%$ at most. This is mainly because the higher the mass fraction, the more intense the Brownian motion for nanoparticles. The strong Brownian motion can destroy the thermal boundary layer generated in the forced convection process. Therefore, the thermal resistance caused by thermal boundary layer is weakened. 
Furthermore, nanoparticles also increase the overall thermal conductivity, and then

456

457

458

459

460

461

462

463

464

465

466

467

improve heat transfer effect of nanofluids. Meanwhile, from Figs. 10-12, it is not

difficult to find that heat transfer intensity of turbulent flow is significantly higher

than laminar flow. This is mainly due to the slow flow velocity and weak convective

heat transfer capacity in laminar flow, and in this case, heat conduction dominates the

heat transfer. However, as the flow velocity augments, the convective heat transfer

capacity is gradually improved, and irregular movement of nanoparticles is gradually

intensified, which can further damage the thermal boundary layer, as well as improve

the heat transfer.
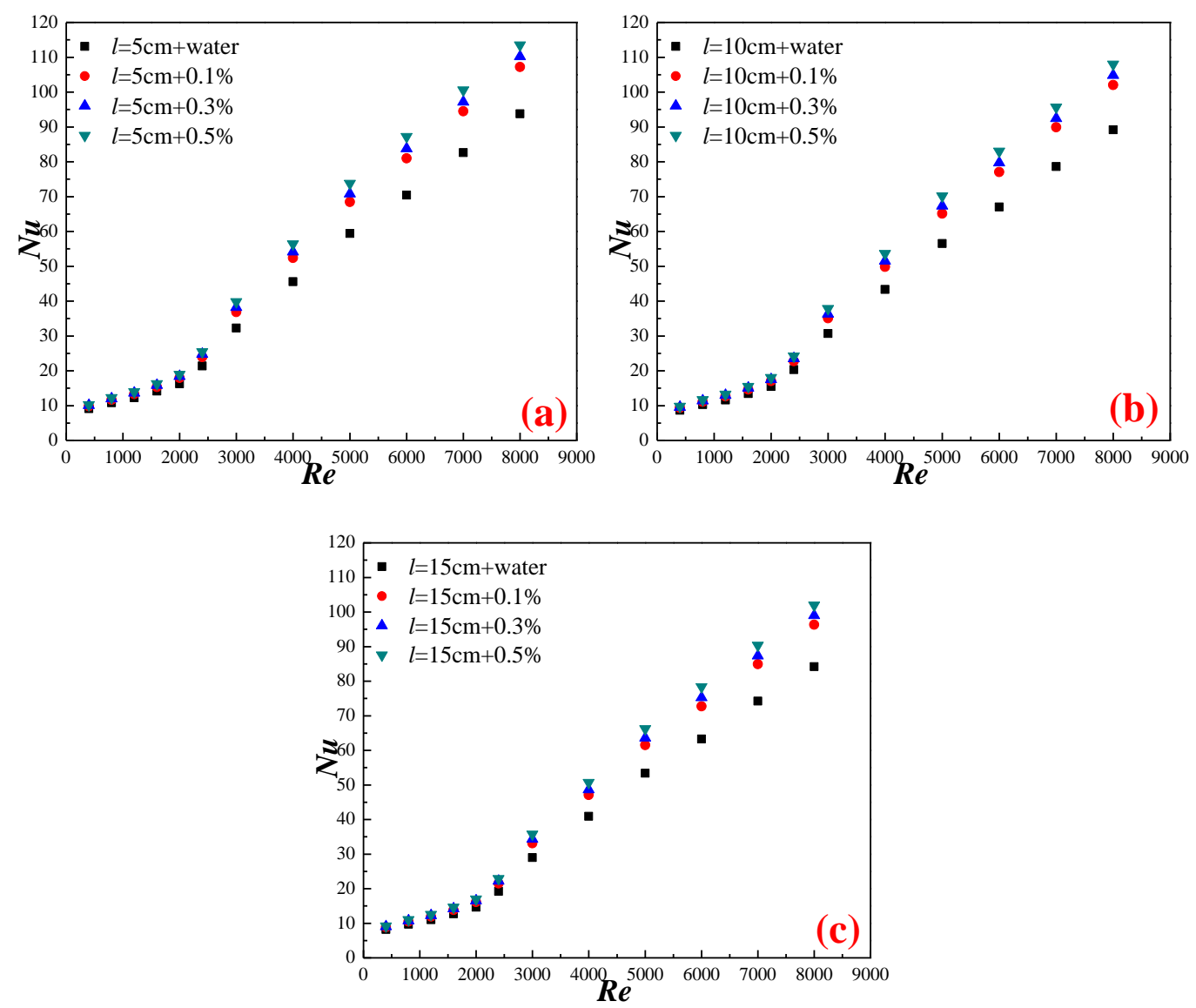

Fig. 12. Effects of nanoparticle mass fractions on Nusselt numbers of triangular tube, $d=5 \mathrm{~mm}$ : (a) $l=5 \mathrm{~cm}$, (b) $l=10 \mathrm{~cm}$, (c) $l=15 \mathrm{~cm}$ 


\subsubsection{Effect of round hole diameter}

Effects of round hole diameter $(d=3 \mathrm{~mm}, d=4 \mathrm{~mm}, d=5 \mathrm{~mm})$ on the heat transfer characteristics of the working fluids in the triangular tube with perforated turbulators and mass fractions of nanoparticles are researched, too. From Figs. 13-15, it is observed that $d=5 \mathrm{~mm}$ exhibits the superior heat transfer capacity.
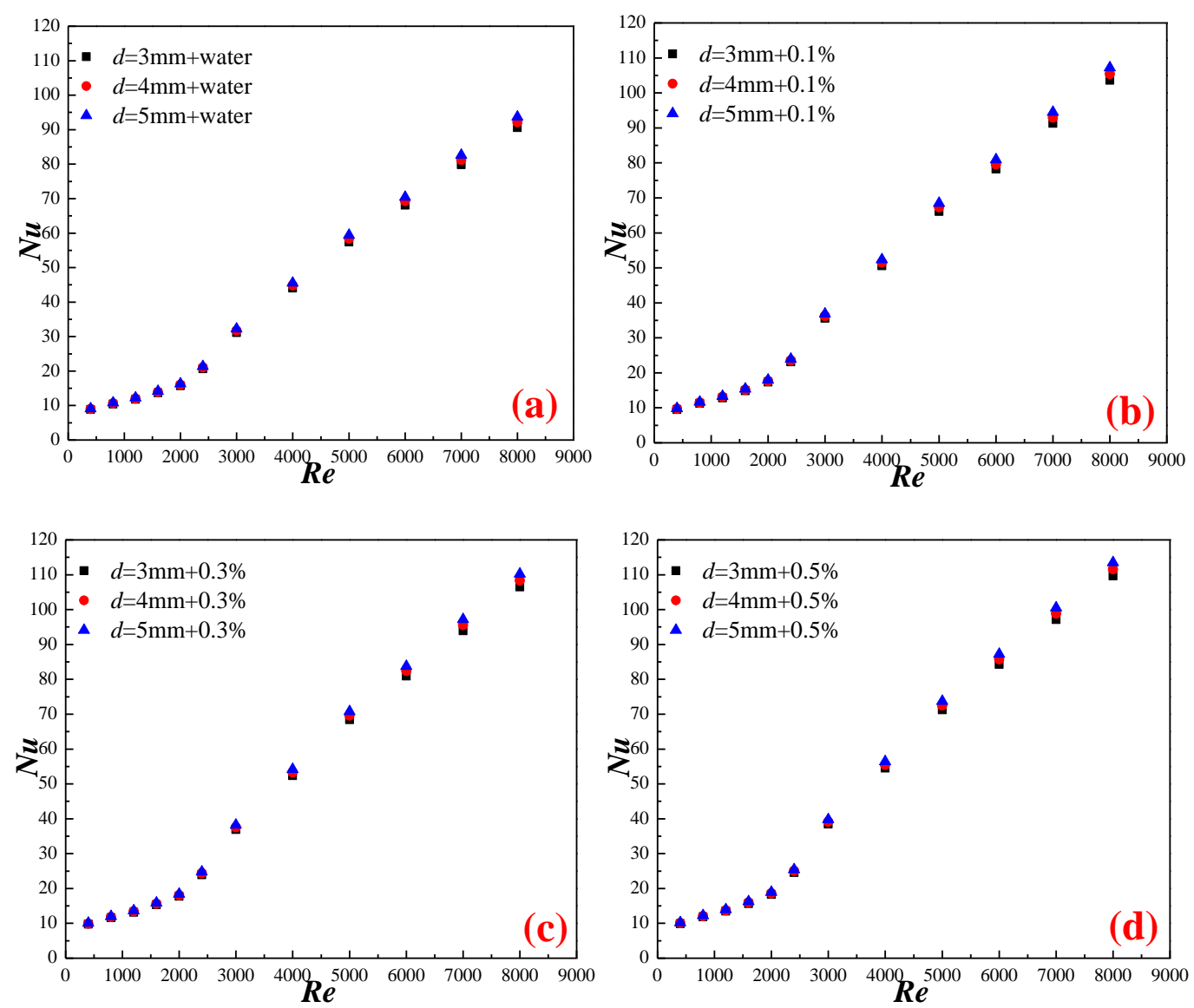

Fig. 13. Effects of hole diameters on Nusselt numbers of triangular tube, $l=5 \mathrm{~cm}$ : (a) $\omega=0 \%$, (b) $\omega=0.1 \%$, (c) $\omega=0.3 \%$, (d) $\omega=0.5 \%$

For the triangular tube with a perforated turbulator of round holes pitch-row $l=5 \mathrm{~cm}$ in Fig. 13, nanofluids with various mass fractions $(\omega=0 \%, 0.1 \%, 0.3 \%, 0.5 \%)$ can improve the heat transfer by $6.478 \%, 6.424 \%, 6.456 \%, 6.498 \%$ at best when $d=5 \mathrm{~mm}$ compared with that when $d=3 \mathrm{~mm}$ respectively. For the triangular tube with a perforated turbulator of round holes pitch-row $l=10 \mathrm{~cm}$ in Fig. 14, it can enhance the 

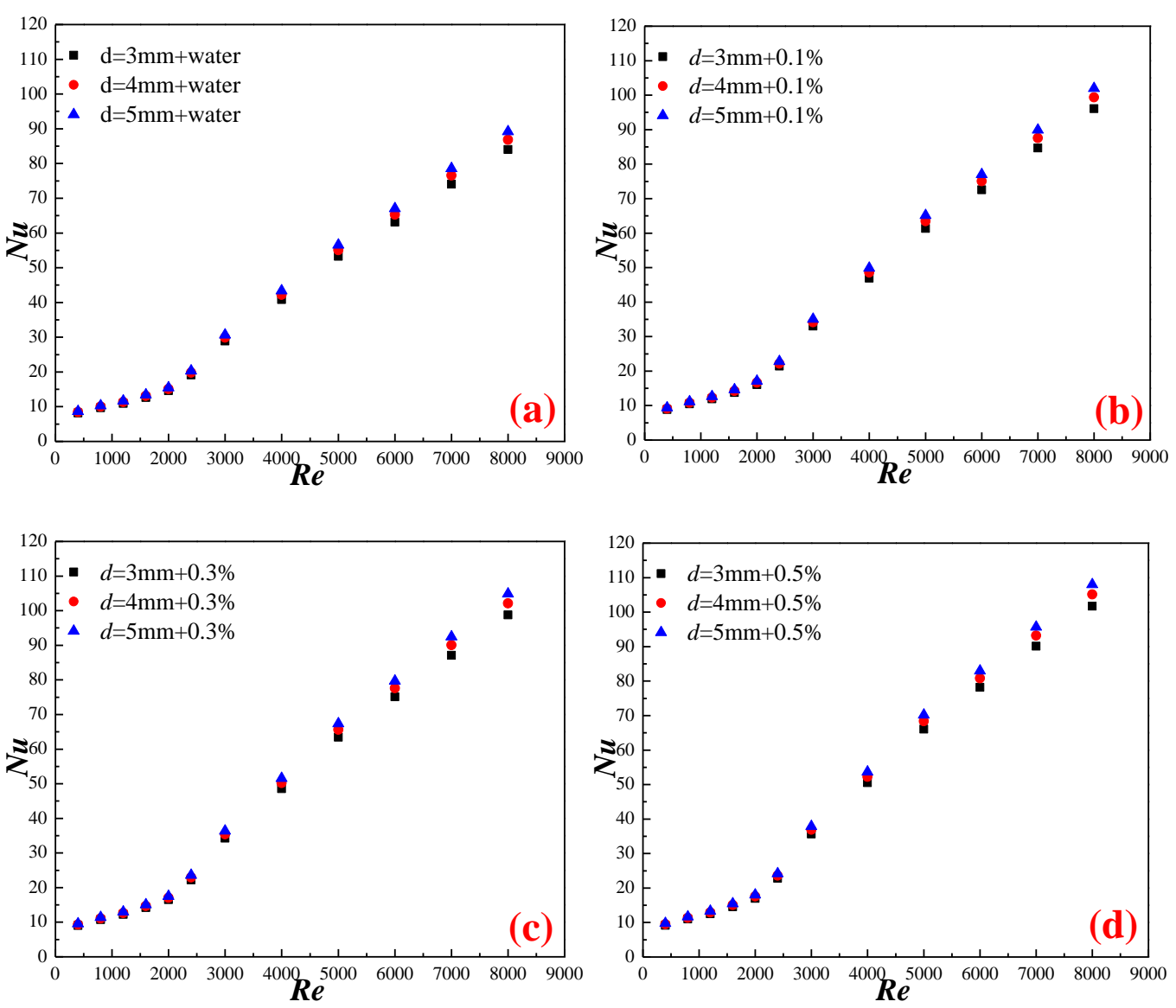

490

Fig. 14. Effects of hole diameters on Nusselt numbers of triangular tube, $l=10 \mathrm{~cm}$ : (a) 491 $\omega=0 \%$, (b) (b) $\omega=0.1 \%$, (c) (c) $\omega=0.3 \%$, (d) $\omega=0.5 \%$

Therefore, the turbulator with the largest diameter round hole $(d=5 \mathrm{~mm})$ can augment the heat transfer by $6.498 \%$ at most in comparison to the turbulator with $d=3 \mathrm{~mm}$. This is because when the aperture ratio of the turbulator is at a low level (the maximum of aperture ratio in this paper is less than $5 \%$, which is in accordance with the concept of low aperture ratio), the holes on the turbulator can promote heat 
transfer. As mentioned above, the turbulator causes the flowing fluid to form a rotating flow and a secondary flow, and then promotes heat transfer. However, for a turbulator with round holes, it increases the turbulence of the rotating flow, so that the disturbance of the wall boundary layer is more obvious. On the other hand, it makes the trajectory of the mainstream particles more complicated, which strengthens the secondary flow. Therefore, when the aperture ratio is low, the larger hole diameter leads to the larger the influence range of the hole and the more obvious the influence on the rotating flow and the secondary flow, that is, the heat transfer can be promoted.
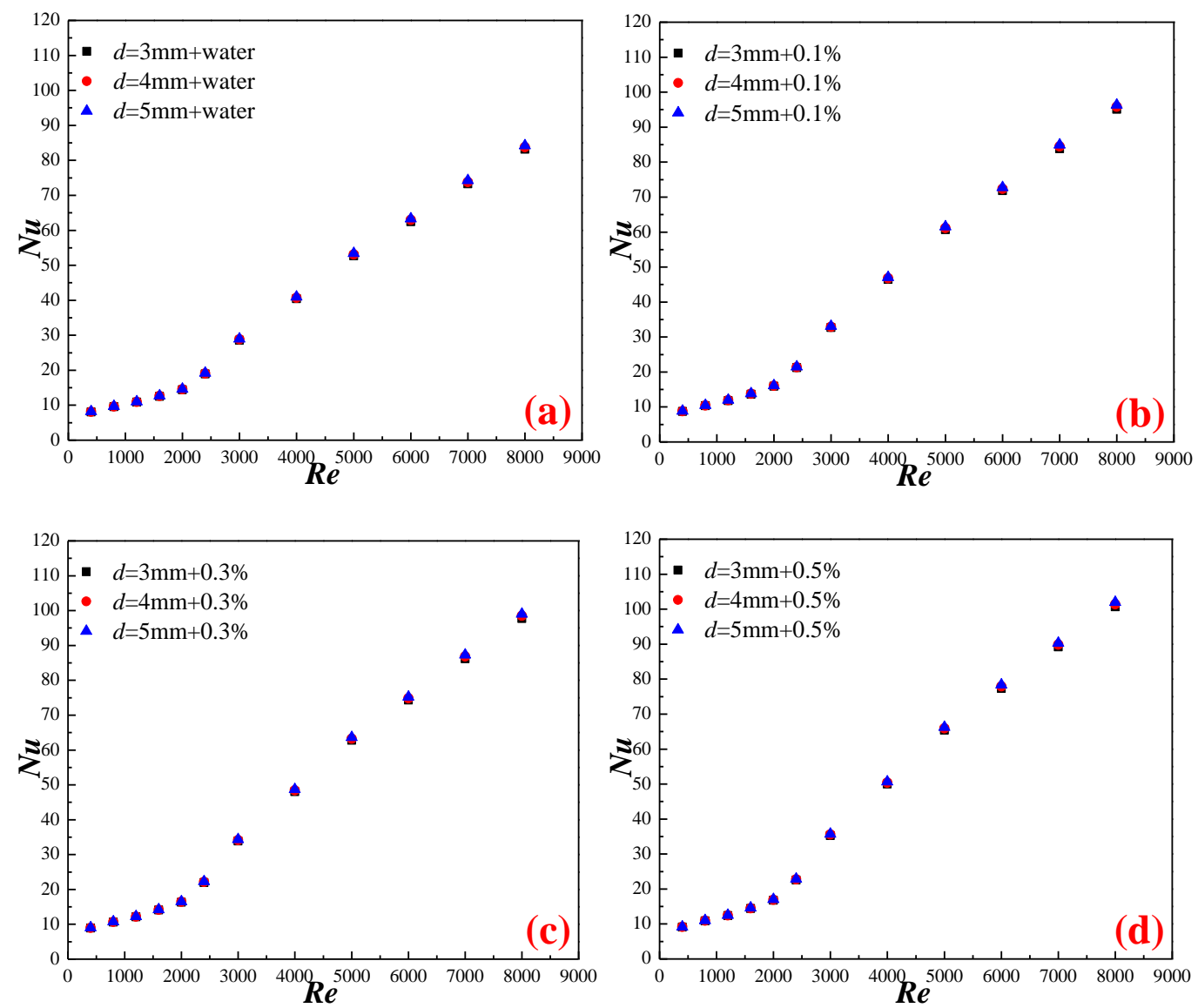

Fig. 15. Effects of hole diameters on Nusselt numbers of triangular tube, $l=15 \mathrm{~cm}$ : (a) $\omega=0 \%$, (b) $\omega=0.1 \%$, (c) $\omega=0.3 \%$, (d) $\omega=0.5 \%$

\subsubsection{Effect of round hole pitch-row}

Similarly, effects of round hole pitch-rows $(l=5 \mathrm{~cm}, l=10 \mathrm{~cm}, l=15 \mathrm{~cm})$ on heat 
Nusselt number with different nanoparticle mass fractions $(\omega=0 \%, 0.1 \%, 0.3 \%, 0.5 \%)$.

It is found that the triangular tube with turbulators containing a round hole pitch-row pitch-row for heat transfer enhancement is similar to that of round hole diameter.

When the aperture ratio is low, the smaller the hole pitch-row leads to the larger and the secondary flow, that is, the heat transfer can be promoted.
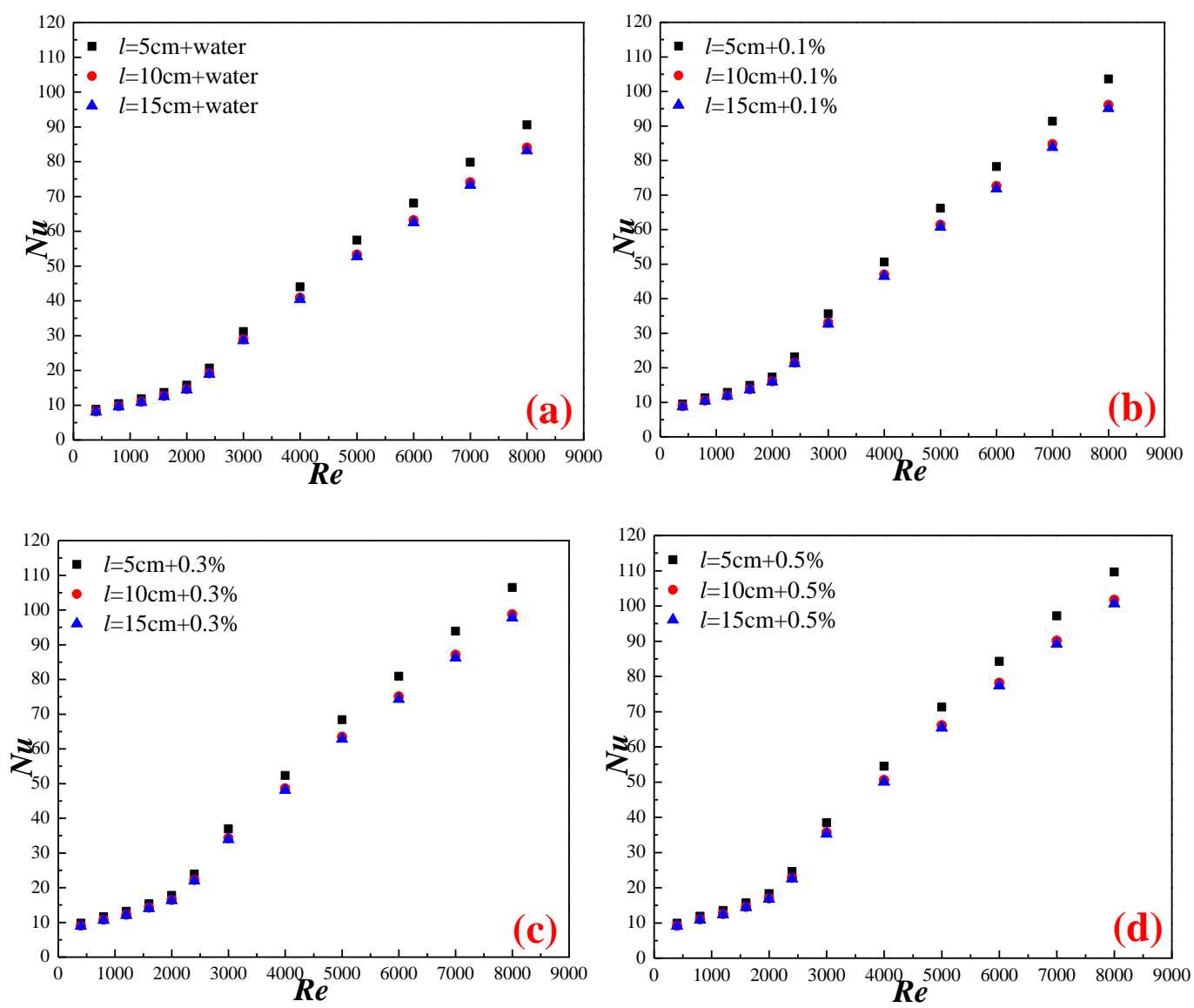

Fig. 16. Effects of hole pitch-row on Nusselt numbers of triangular tube, $d=3 \mathrm{~mm}$ : (a) $\omega=0 \%$, (b) $\omega=0.1 \%$, (c) $\omega=0.3 \%$, (d) $\omega=0.5 \%$ 

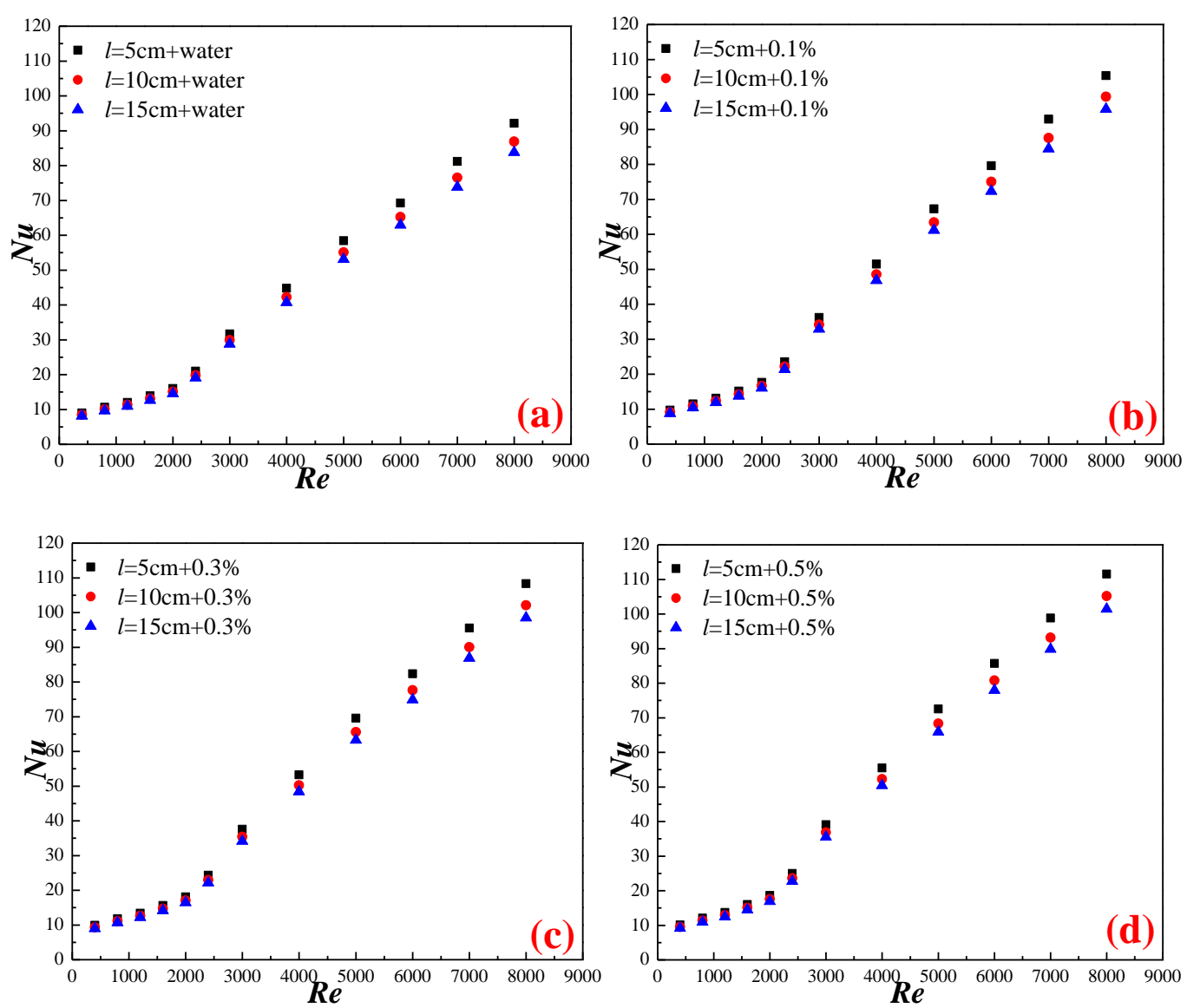

Fig. 17. Effects of hole pitch-row on Nusselt numbers of triangular tube, $d=4 \mathrm{~mm}$ : (a) $\omega=0 \%$, (b) $\omega=0.1 \%$, (c) $\omega=0.3 \%$, (d) $\omega=0.5 \%$ 

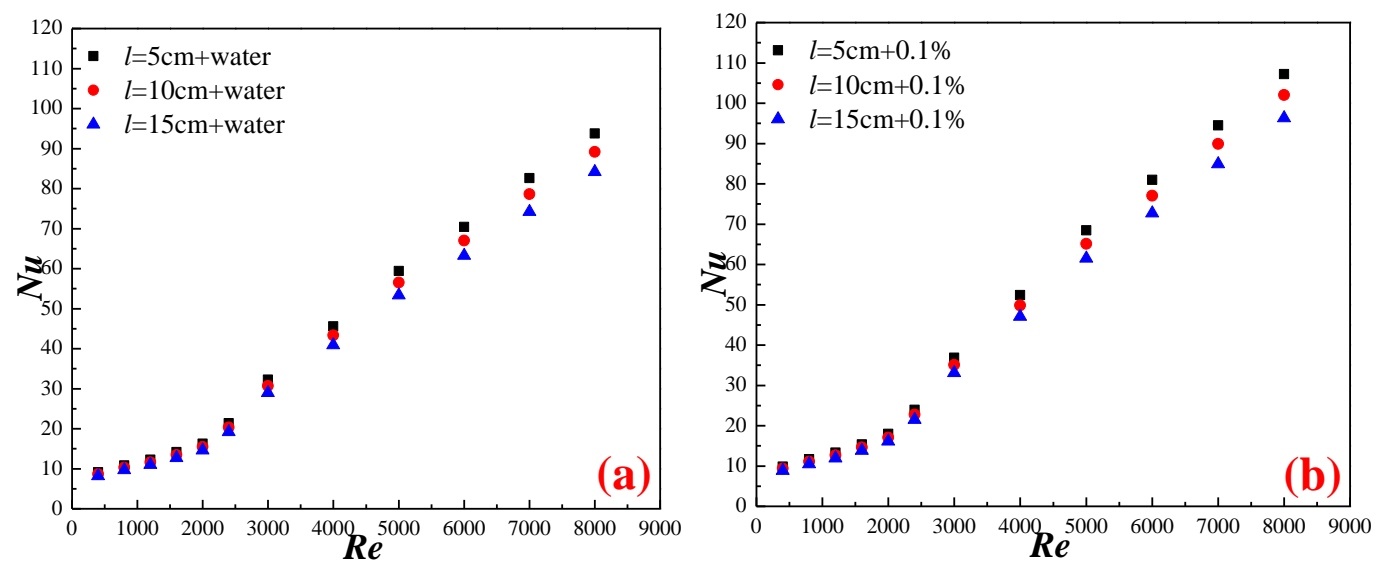

540

541

542

543

544
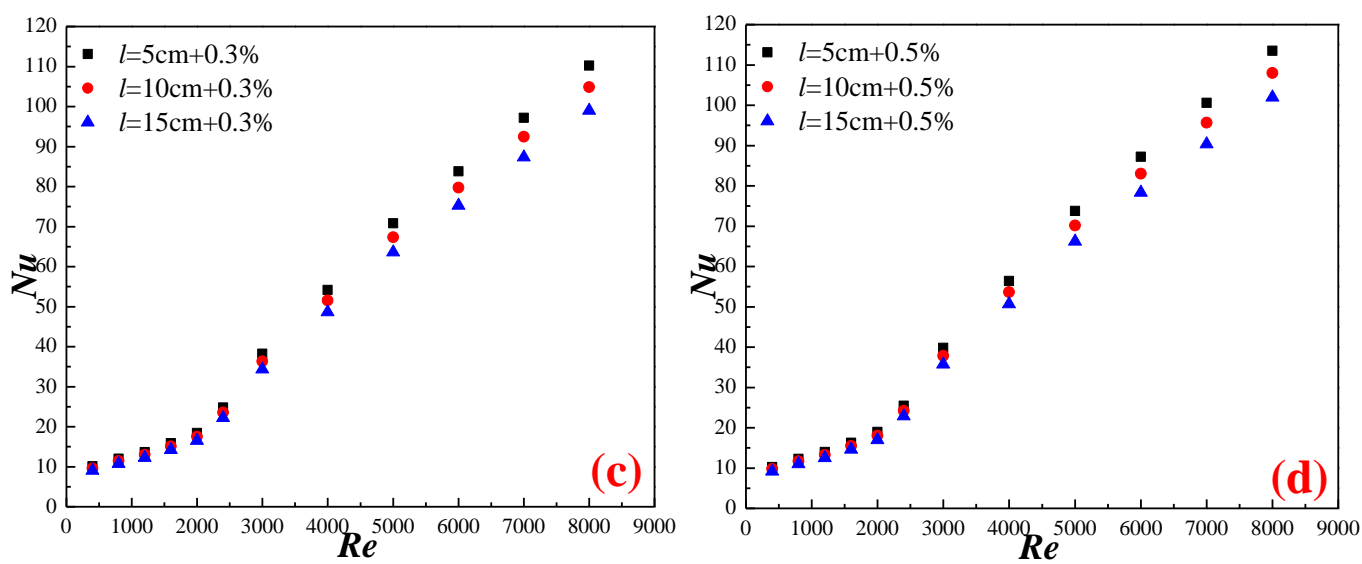

Fig. 18. Effects of hole pitch-row on Nusselt numbers of triangular tube, $d=5 \mathrm{~mm}$ : (a) $\omega=0 \%$, (b) $\omega=0.1 \%$, (c) $\omega=0.3 \%$, (d) $\omega=0.5 \%$

hole pitch-row $l=15 \mathrm{~cm}$ when $\omega=0 \%, 0.1 \%, 0.3 \%, 0.5 \%$.

\subsubsection{Resistance coefficient}

Regardless of nanofluids or turbulators, not only the heat transfer capacity is improved, but also the flow resistance is affected by them. Compared with the single triangular tube in the Fig. 19, the insertion of the turbulator has a greater influence on the flow characteristics. When the mass fractions are $0.0 \mathrm{wt} \%, 0.1 \mathrm{wt} \%, 0.3 \mathrm{wt} \%$ and $0.5 \mathrm{wt} \%$, the flow resistance can be increased by $19.34 \%, 20.49 \%, 22.25 \%$, and $22.28 \%$ respectively. The following is an explanation for this phenomenon. From the previous analysis, it has been known that turbulators cause the fluid to produce a rotating flow and a secondary flow. These two kinds of flow not only improve the heat transfer 
553

554

555

556

557

558

559

560

561

562

563

564

effect, but also increase the flow resistance. The rotating flow increases the flow path of the fluid and exacerbates the friction of the fluid with the tube wall and the turbulator. Besides, the probability of collision between the nanoparticles and the tube wall is also increased by the rotating flow. And the secondary flow intensifies the collision of the fluid itself, and results in a loss of kinetic energy of water molecules and nanoparticles, which causes the increase of flow resistance at the macroscopic level.

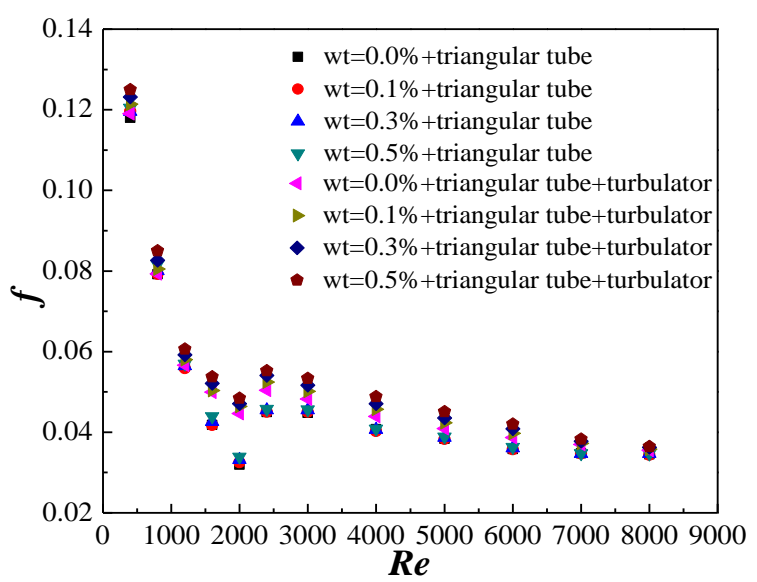

Fig. 19 Changes of flow resistance $f$ of $\mathrm{SiO}_{2}-\mathrm{H}_{2} \mathrm{O}$ nanofluids with $R e$ in the triangular tube with/without turbulator

The relation between mass fraction of nanoparticles and resistance coefficient is also explored in this paper. This relation is revealed in Fig. 20 based on the triangular tube with perforated turbulators (round holes diameter $d=3 \mathrm{~mm}, d=4 \mathrm{~mm}$ and $d=5 \mathrm{~mm}$ ). It can be noted that as the mass fraction augments, the resistance coefficient increases gradually. However, due to the different densities between nanoparticles and water, there is a disparity between them in the velocity of flow, which leads to the generation of resistance. In addition, as the augment of nanoparticles mass percentage, the liquid viscosity increases and leads to an increase in resistance coefficient. Therefore, a great resistance coefficient is mainly caused by the large viscosity. 

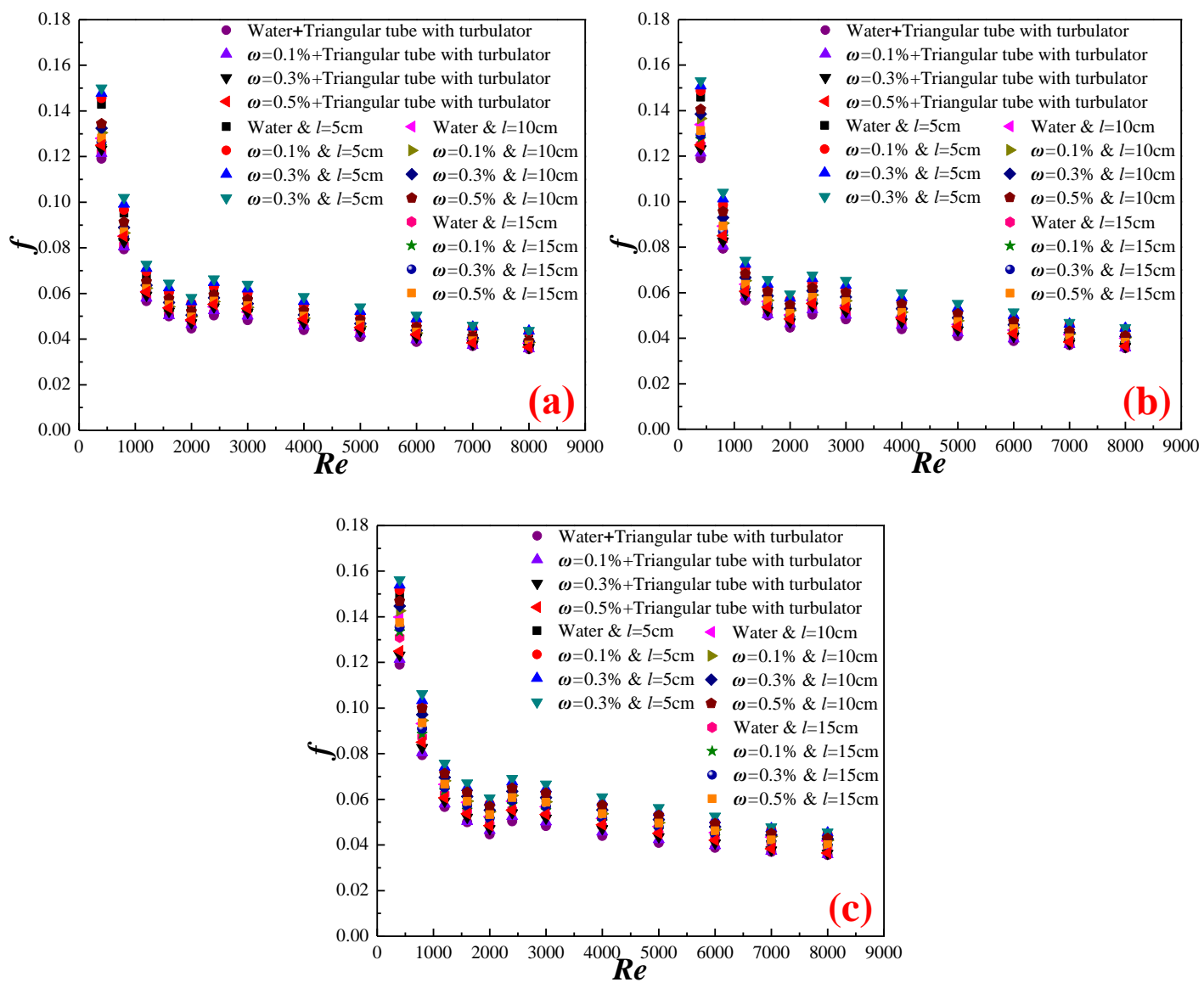

574

Fig. 20. Changes of resistance coefficient with Reynolds numbers, (a) $d=3 \mathrm{~mm}$, (b)

$$
d=4 \mathrm{~mm}, \text { (c) } d=5 \mathrm{~mm}
$$

\subsubsection{Effect of nanoparticle mass fraction}

From Figs. 21-23, it can be found as follows: For the triangular tube with a perforated turbulator (round holes diameter $d=3 \mathrm{~mm}$ ), nanofluids with $\omega=0.5 \%$ can bring about the increase of resistance coefficient by $10.193 \%, 10.503 \%, 11.230 \%$ at most compared with D-I water when $l=5 \mathrm{~cm}, 10 \mathrm{~cm}, 15 \mathrm{~cm}$ respectively. For the triangular tube with a perforated turbulator (round holes diameter $d=4 \mathrm{~mm}$ ), nanofluids with $\omega=0.5 \%$ can bring about the increase of resistance coefficient by $10.458 \%, 10.763 \%, 11.375 \%$ at most compared with D-I water when $l=5 \mathrm{~cm}, 10 \mathrm{~cm}$, $15 \mathrm{~cm}$ respectively. For the triangular tube with a perforated turbulator (round holes diameter $d=5 \mathrm{~mm}$ ), nanofluids with $\omega=0.5 \%$ can bring about the increase of 

water when $l=5 \mathrm{~cm}, 10 \mathrm{~cm}, 15 \mathrm{~cm}$ respectively.

These phenomena are mainly caused by two factors: Firstly, as mentioned above,

589 the mass difference between nanoparticles and water molecules results in the velocity

590 difference when they flow, and the velocity difference increases the friction, and lastly

591 increases the viscosity. Secondly, flow in the tube is forced convection, and

592 nanoparticles will aggravate the friction between fluid and tube, and thereby increase

593 the resistance coefficient. In the meantime, the higher the mass percentage is, the 594 more obvious the enhancement effect of above two factors on the resistance 595 coefficient can be.
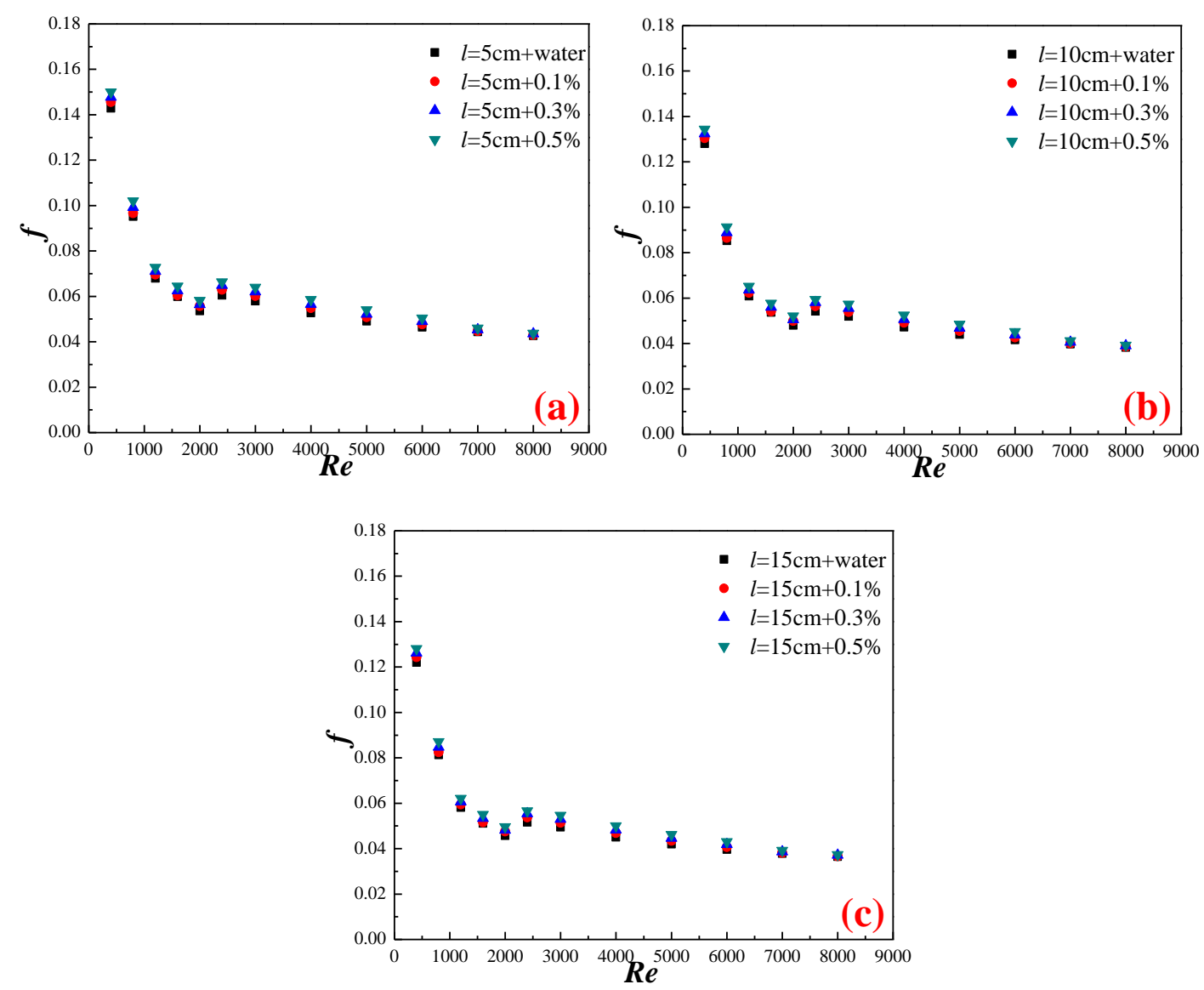

Fig. 21. Effects of nanoparticle mass fractions on resistance coefficient of triangular tube, $d=3 \mathrm{~mm}$ : (a) $l=5 \mathrm{~cm}$, (b) $l=10 \mathrm{~cm}$, (c) $l=15 \mathrm{~cm}$ 

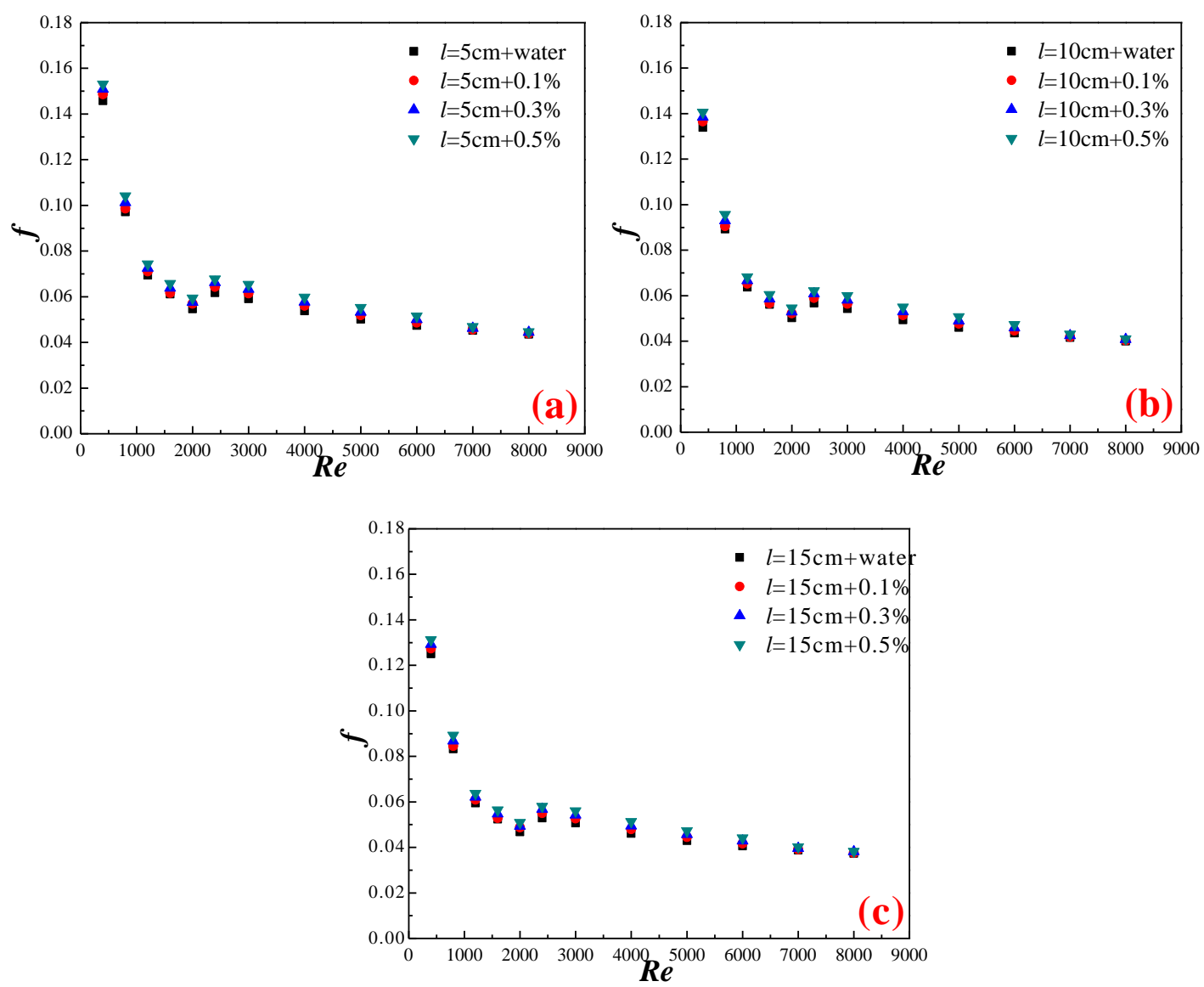

Fig. 22. Effects of nanoparticle mass fractions on resistance coefficient of triangular tube, $d=4 \mathrm{~mm}$ : (a) $l=5 \mathrm{~cm}$, (b) $l=10 \mathrm{~cm}$, (c) $l=15 \mathrm{~cm}$
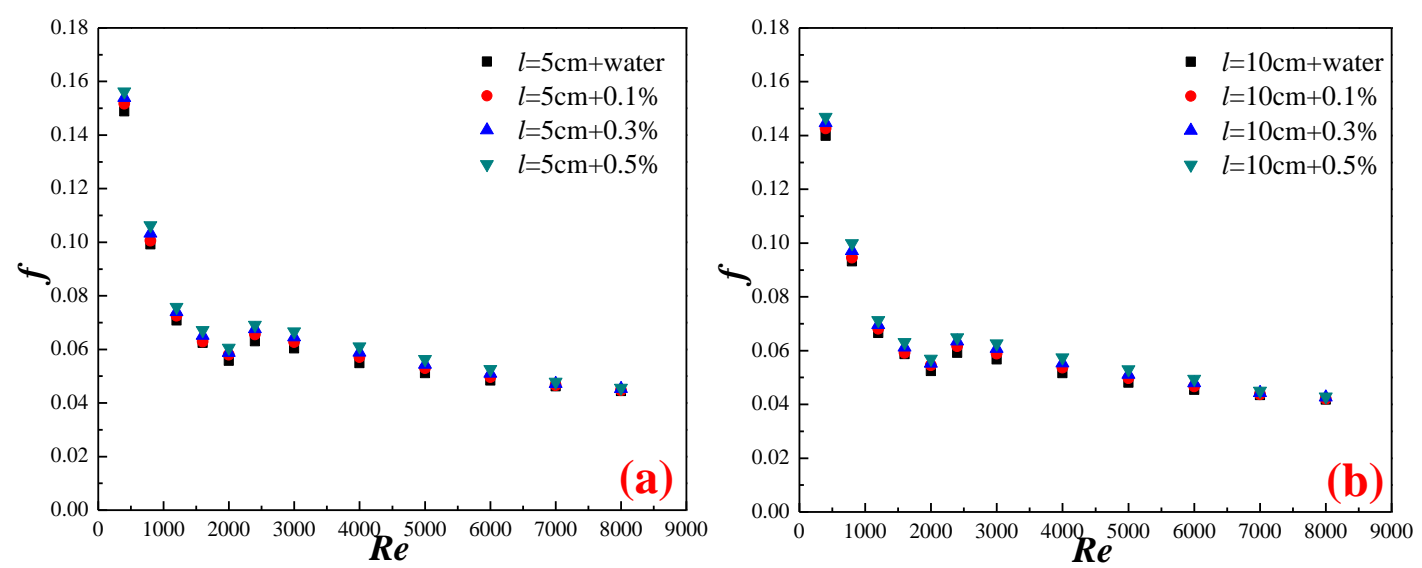
605

606

607

608

609

610

611

612

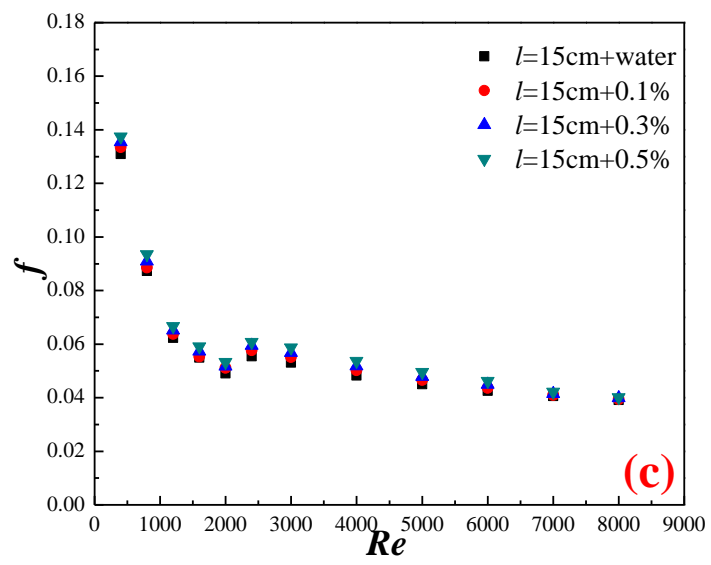

Fig. 23. Effects of nanoparticle mass fractions on resistance coefficient of triangular tube, $d=5 \mathrm{~mm}$ : (a) $l=5 \mathrm{~cm}$, (b) $l=10 \mathrm{~cm}$, (c) $l=15 \mathrm{~cm}$

\subsubsection{Effect of round hole diameter}

Effects of round hole diameter on the flow performance are investigated. From Figs. 24, 25 and 26, it can be discovered that the hole diameters $(d=3 \mathrm{~mm}, d=4 \mathrm{~mm}$, $d=5 \mathrm{~mm}$ ) have an impact on the resistance coefficient. When the fluid flows in a tube with a turbulator, it produces a rotating flow and a secondary flow, and both of them affect the increase of the flow resistance. Their specific mechanism of affecting flow resistance has already been mentioned. For a turbulator with round holes, when the aperture ratio is at a low level, these holes strengthen the intensity of the rotating flow and the secondary flow generated by the turbulator, and then make the flow resistance larger. And as the hole diameter increases, the range of influence of the hole will also increase, and accordingly, the increase in flow resistance will be more obvious.

For the triangular tube with a perforated turbulator (round holes pitch-row $l=5 \mathrm{~cm}$ ), the holes diameter $d=5 \mathrm{~mm}$ can bring about the increase of resistance coefficient by $9.102 \%, 9.188 \%, 9.274 \%, 9.302 \%$ at most when $\omega=0 \%, 0.1 \%, 0.3 \%$, $0.5 \%$ respectively. For the triangular tube with a perforated turbulator (round holes pitch-row $l=10 \mathrm{~cm}$ ), the holes diameter $d=5 \mathrm{~mm}$ can bring about the increase of 
624

resistance coefficient by $8.881 \%, 8.932 \%, 8.947 \%, 8.981 \%$ at most when $\omega=0 \%$, $0.1 \%, 0.3 \%, 0.5 \%$ respectively. For the triangular tube with a perforated turbulator (round holes pitch-row $l=15 \mathrm{~cm}$ ), the holes diameter $d=5 \mathrm{~mm}$ can bring about the increase of resistance coefficient by $8.450 \%, 8.579 \%, 8.704 \%, 8.794 \%$ at most when $\omega=0 \%, 0.1 \%, 0.3 \%, 0.5 \%$ respectively.
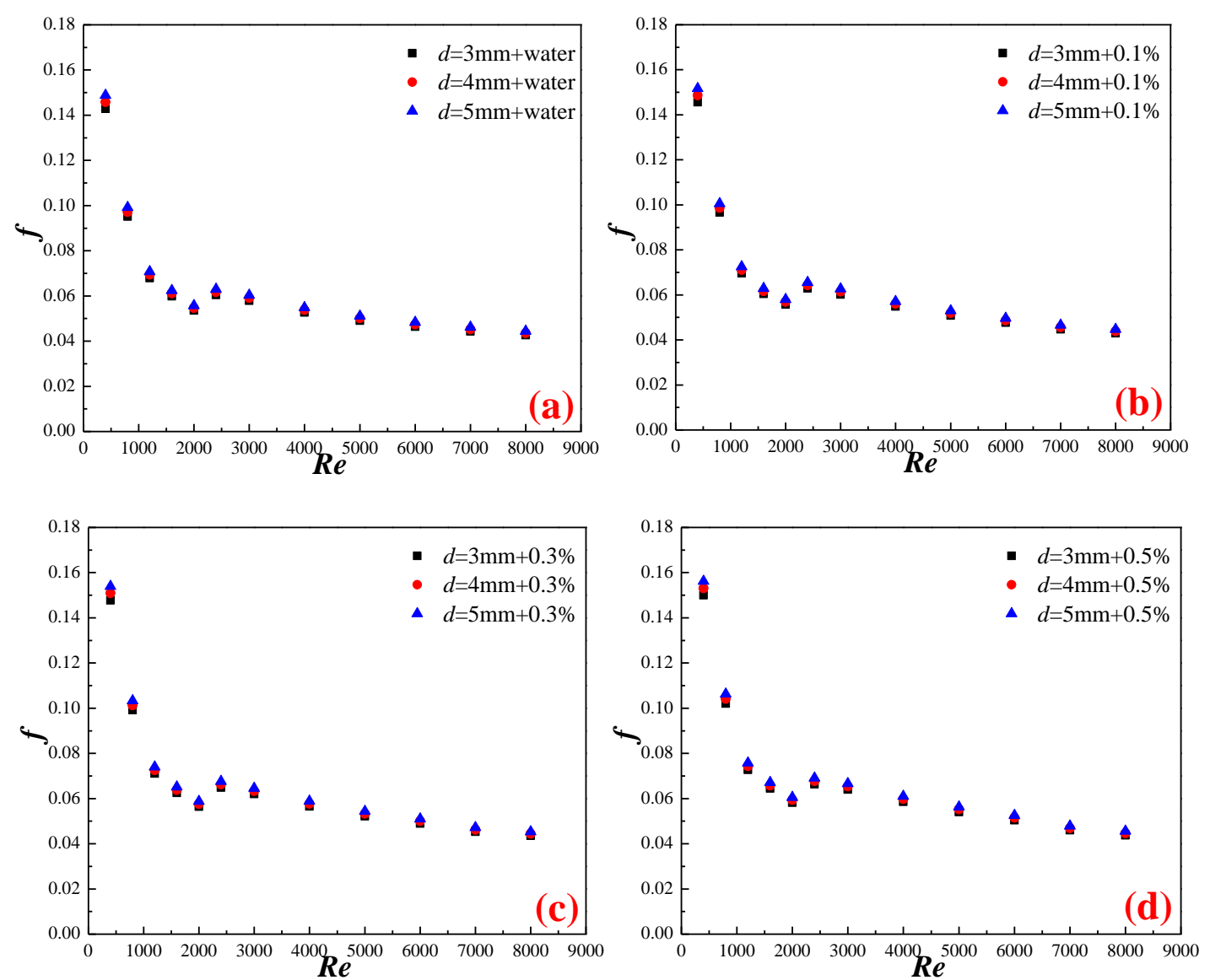

Fig. 24. Effects of hole diameters on resistance coefficient of triangular tube, $l=5 \mathrm{~cm}$ : (a) $\omega=0 \%$, (b) $\omega=0.1 \%$, (c) $\omega=0.3 \%$, (d) $\omega=0.5 \%$
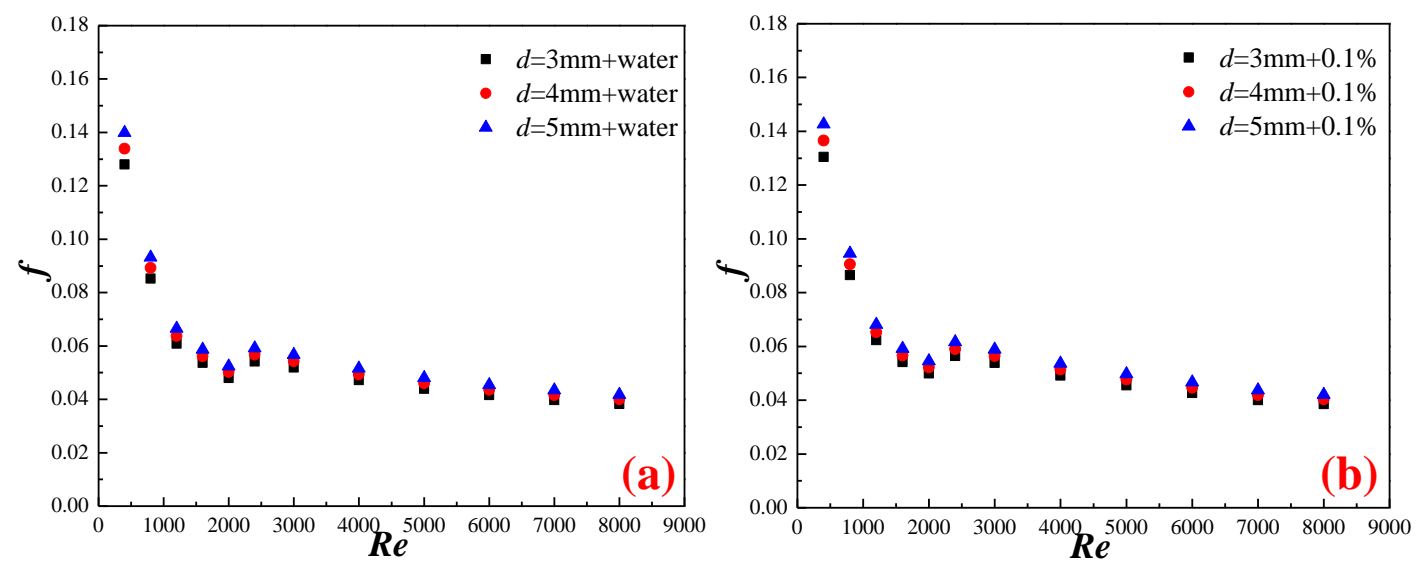
634

635
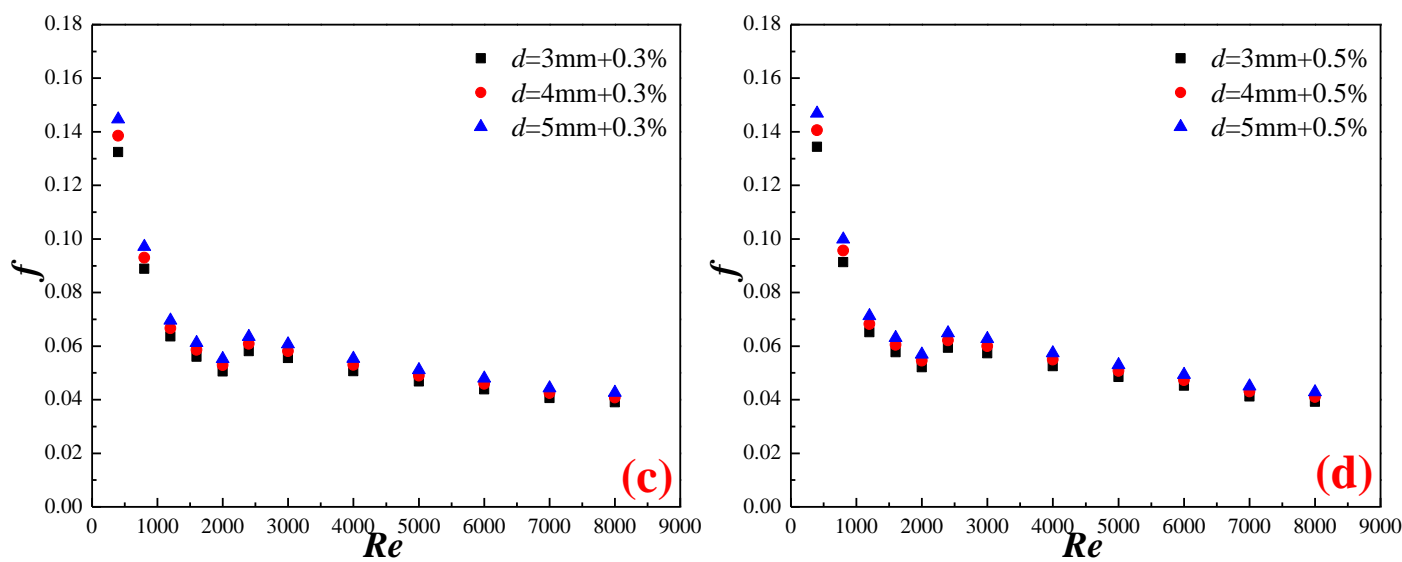

Fig. 25. Effects of hole diameters on resistance coefficient of triangular tube, $l=10 \mathrm{~cm}$ : (a) $\omega=0 \%$, (b) $\omega=0.1 \%$, (c) $\omega=0.3 \%$, (d) $\omega=0.5 \%$
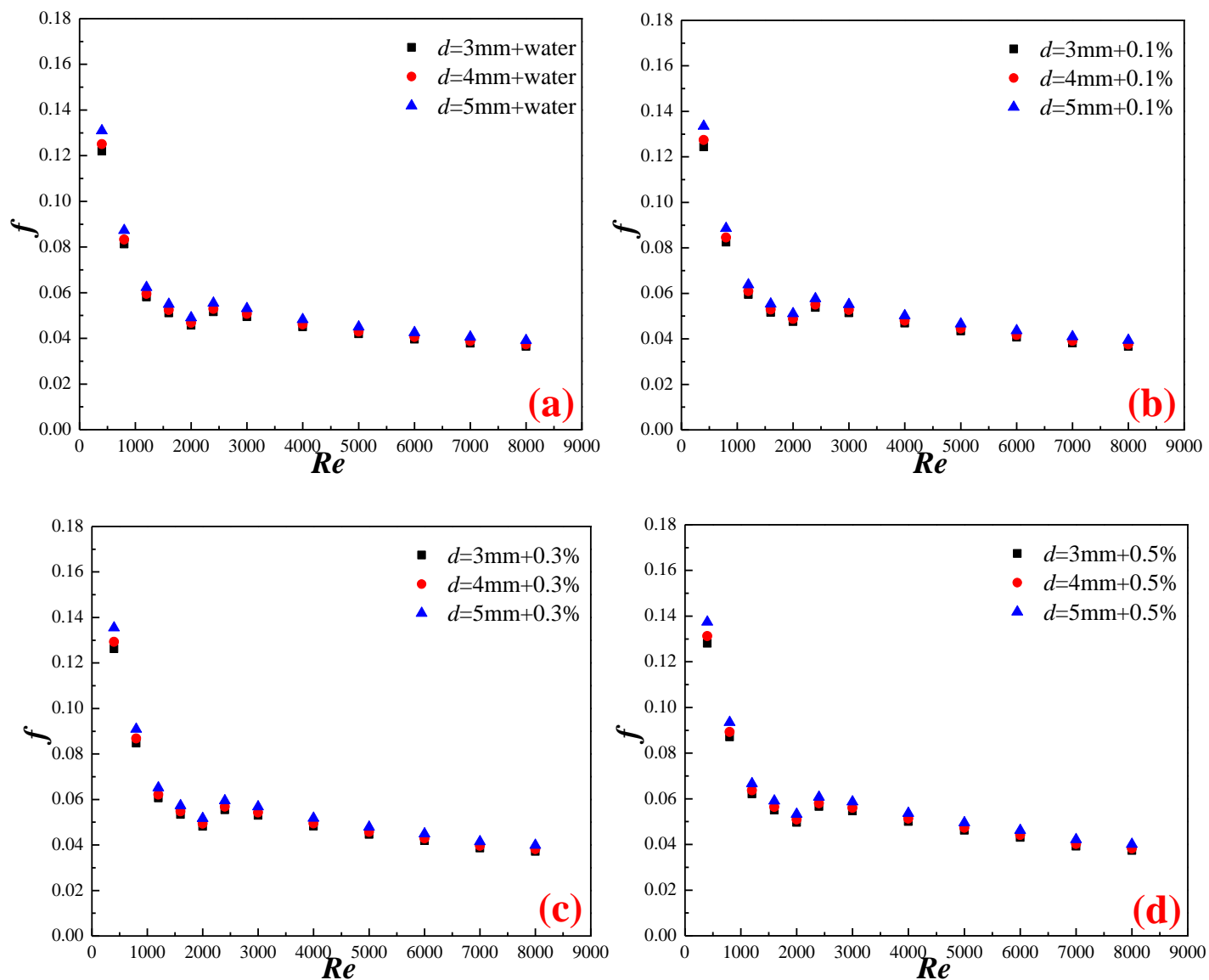

Fig. 26. Effects of hole diameters on resistance coefficient of triangular tube, $l=15 \mathrm{~cm}$ : (a) $\omega=0 \%$, (b) $\omega=0.1 \%$, (c) $\omega=0.3 \%$, (d) $\omega=0.5 \%$

\subsubsection{Effect of round hole pitch-row}

Effects of round hole pitch-rows $(l=5 \mathrm{~cm}, l=10 \mathrm{~cm}, l=15 \mathrm{~cm})$ on the flow performance are also studied. Figs. 27, 28 and 29 show the effects of round hole pitch-row on the resistance coefficient with different nanoparticle mass fractions 
645

646

647

648

649

650

651

652

653

654

655

656

657

658

659

660

661

662

$(\omega=0 \%, 0.1 \%, 0.3 \%, 0.5 \%)$ respectively. It can be found that triangular tube with a turbulator containing round holes with pitch-row $l=5 \mathrm{~cm}$ exhibits the largest resistance coefficient, which is followed by the round hole pitch-row $l=10 \mathrm{~cm}$, and the triangular tube with a turbulator containing round holes with pitch-row $l=15 \mathrm{~cm}$ exhibits the smallest resistance coefficient. The reason for this experimental phenomenon is similar to round hole diameter. When the aperture ratio is low, the smaller the hole pitch-row, the larger the influence range of the hole, and the more obvious the influence on the rotating flow and the secondary flow, that is, the resistance coefficient can be increased. The specific results are as follows:

With round hole diameter $d=3 \mathrm{~mm}$, the triangular tube with round hole pitch-row $l=5 \mathrm{~cm}$ can increase the resistance coefficient by $10.047 \%, 10.172 \%, 10.236 \%, 10.548 \%$ at best compared with round hole pitch-row $l=15 \mathrm{~cm}$ when $\omega=0 \%, 0.1 \%, 0.3 \%, 0.5 \%$. With round hole diameter $d=4 \mathrm{~mm}$, the triangular tube with round hole pitch-row $l=5 \mathrm{~cm}$ can increase the resistance coefficient by $10.647 \%, 10.912 \%, 11.114 \%, 11.370 \%$ at best compared with round hole pitch-row $l=15 \mathrm{~cm}$ when $\omega=0 \%, 0.1 \%, 0.3 \%, 0.5 \%$. With round hole diameter $d=5 \mathrm{~mm}$, the triangular tube with round hole pitch-row $l=5 \mathrm{~cm}$ can increase the resistance coefficient by $11.279 \%, 11.492 \%, 11.948 \%, 12.400 \%$ at best compared with round hole pitch-row $l=15 \mathrm{~cm}$ when $\omega=0 \%, 0.1 \%, 0.3 \%, 0.5 \%$. 

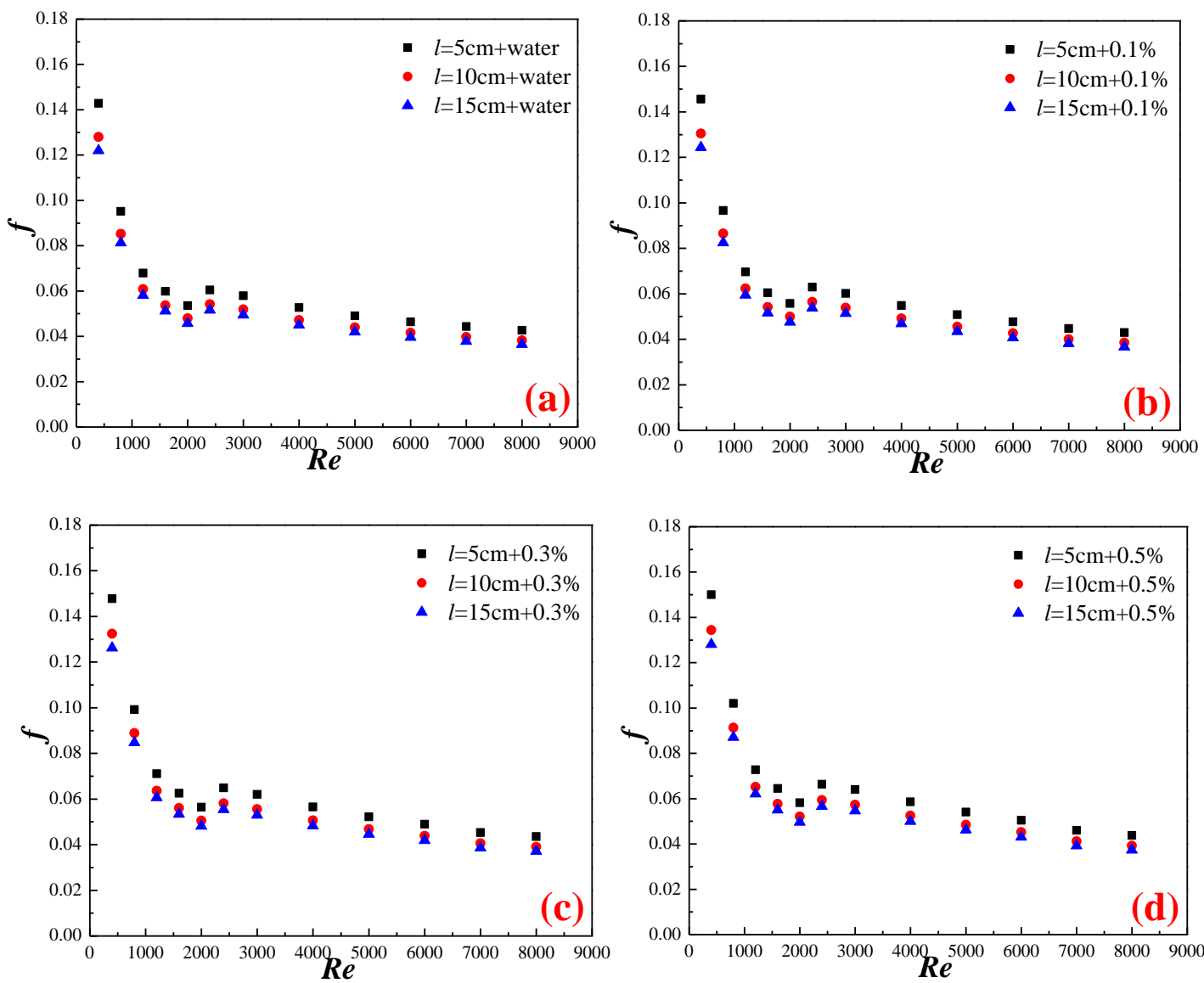

665

Fig. 27. Effects of hole pitch-row on resistance coefficient of triangular tube, $d=3 \mathrm{~mm}$ :

(a) $\omega=0 \%$, (b) $\omega=0.1 \%$, (c) $\omega=0.3 \%$, (d) $\omega=0.5 \%$
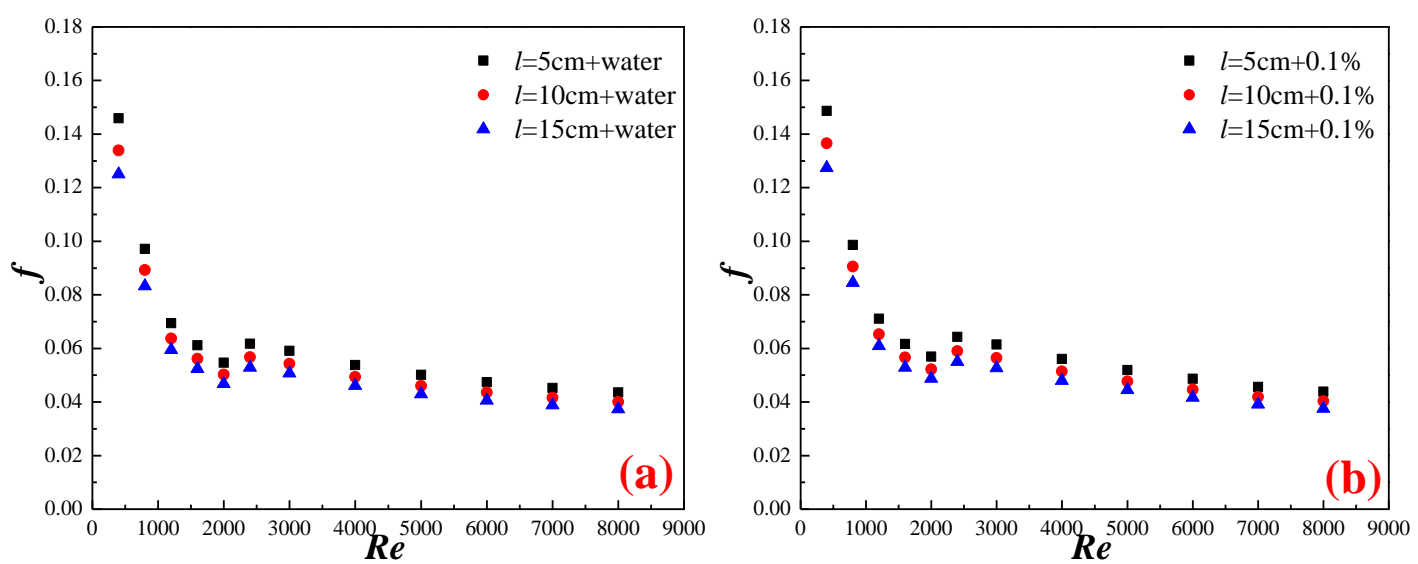
668

669

670

671

672

673

674

675

676

677

678
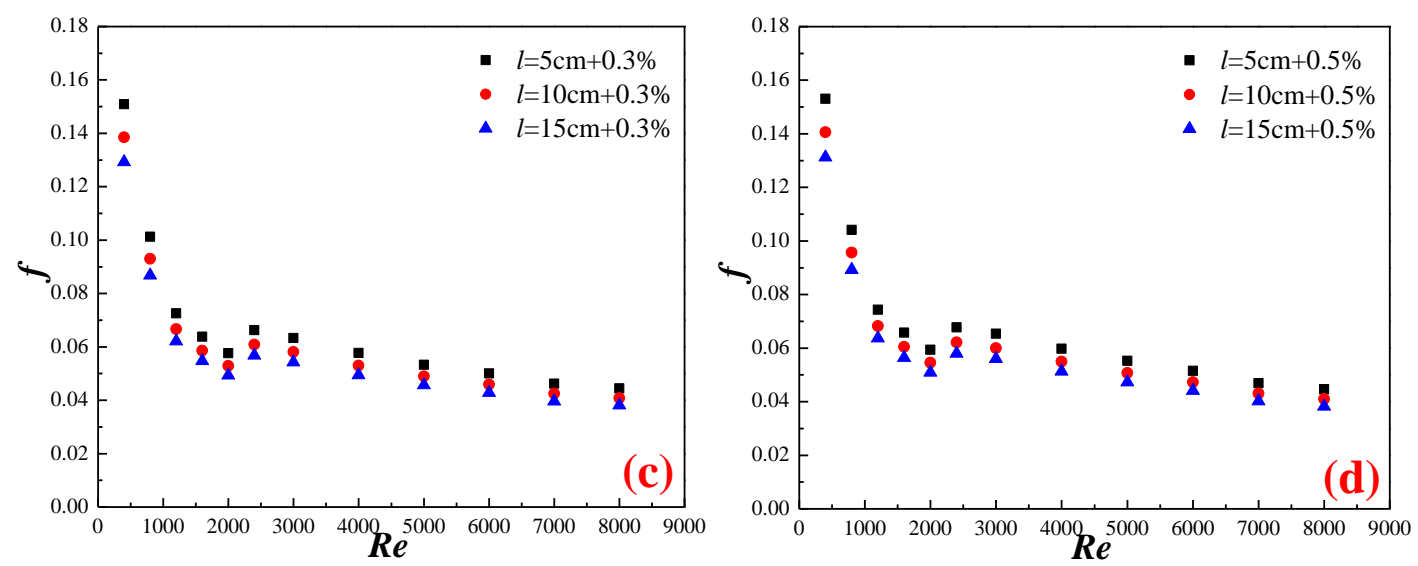

Fig. 28. Effects of hole pitch-row on resistance coefficient of triangular tube, $d=4 \mathrm{~mm}$ : (a) $\omega=0 \%$, (b) $\omega=0.1 \%$, (c) $\omega=0.3 \mathrm{w} \%$, (d) $\omega=0.5 \%$
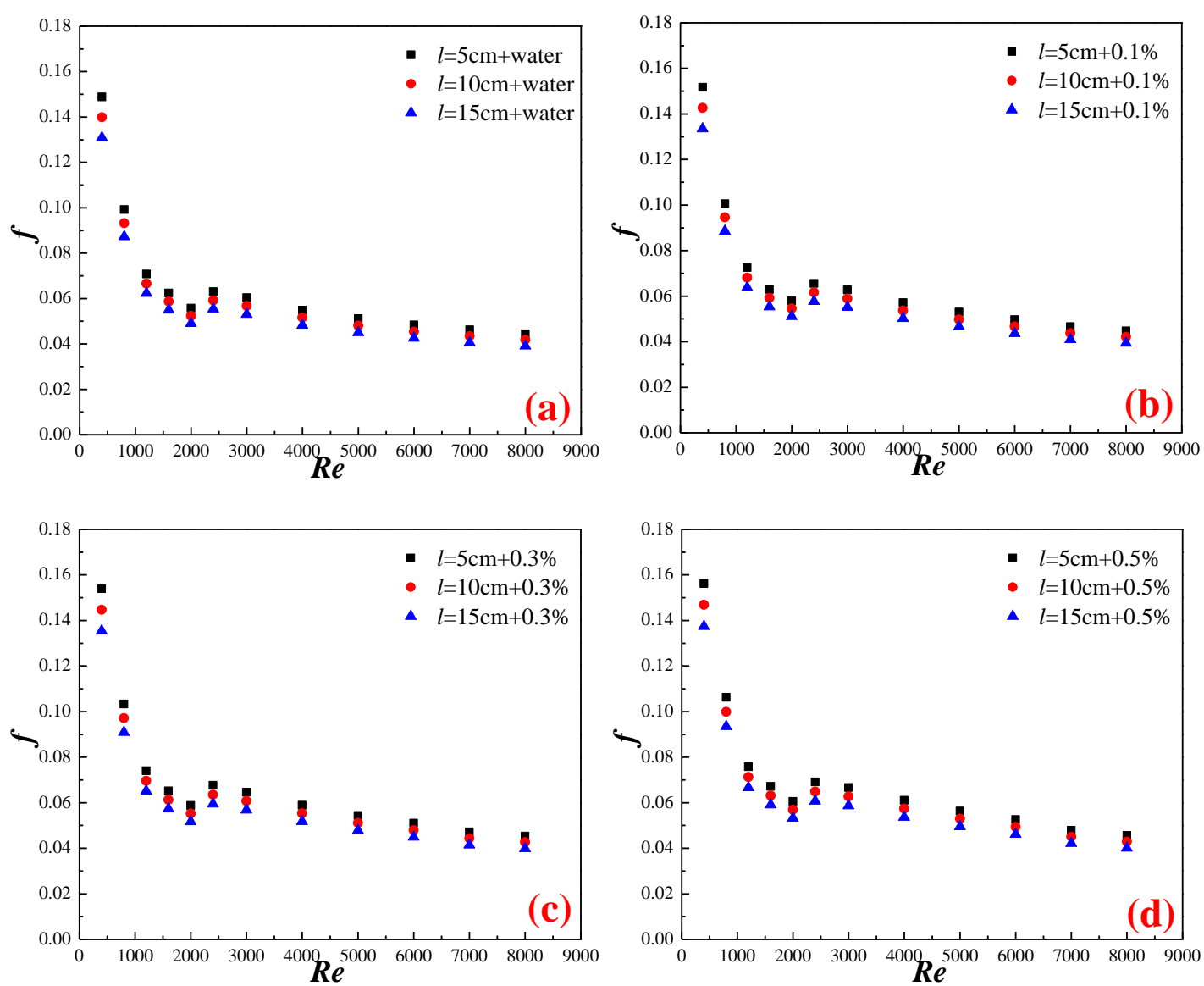

Fig. 29. Effects of hole pitch-row on resistance coefficient of triangular tube, $d=5 \mathrm{~mm}$ : (a) $\omega=0 \%$, (b) $\omega=0.1 \%$, (c) $\omega=0.3 \%$, (d) $\omega=0.5 \%$

\subsubsection{Thermal efficiency evaluation}

According to the above, it can be seen that for one thing, nanoparticles and turbulators in triangular tube can enhance heat transfer. For another, they can bring about a greater pressure drop. In order to consider this experiment in a comprehensive 
679

680

681

682

683

684

685

686

687

688

689

690

691

692

perspective, this paper adopts a comprehensive evaluation index to calculate the experimental data in detail according to the formula (14). The effects of round hole diameter and round hole pitch-row on the comprehensive performance evaluation index are given respectively by Fig. 30 .
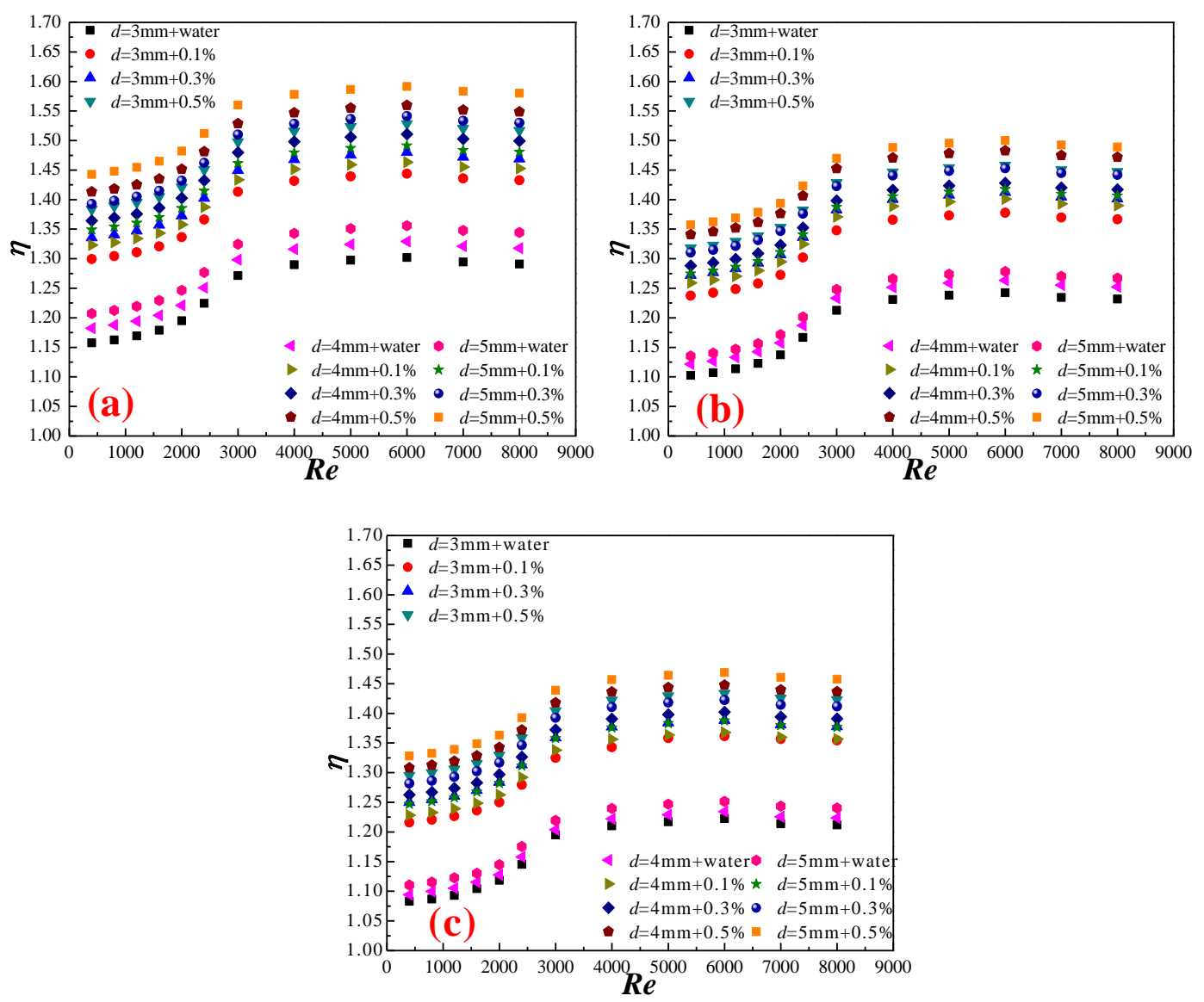

Fig. 30. Effects of round hole diameter on thermal efficiency evaluation index: (a)

$$
l=5 \mathrm{~cm},(\mathrm{~b}) l=10 \mathrm{~cm},(\mathrm{c}) l=15 \mathrm{~cm}
$$

It can be seen from the figure that, the index increases firstly with the increase of $R e$ number, and then decreases with it. The maximum value of this index appears near Reynolds number $R e_{\mathrm{c}}=6000$. When $R e_{\mathrm{c}}<6000$, nanoparticles and the structure would bring about less resistance loss and can effectively enhance the heat transfer. Nevertheless, when $R e_{\mathrm{c}}>6000$, to achieve enhanced heat transfer, more pressure drop needs to be wasted. Besides, these results reveal that the index increases with the 
693

694

695

696

697

698

699

700

701

702

703

704

705

706

707

708

709

710

711

712

713

714

increasing round hole diameter and decreasing round hole pitch-row. It is because that large size and number of round holes can cause higher turbulence and then improve heat transfer. At the same time, the results reveal that the index of $d=5 \mathrm{~mm}$ is the largest one as well as $d=4 \mathrm{~mm}$ follows it, and the index of $d=3 \mathrm{~mm}$ is the smallest. The index of liquids $(\omega=0.5 \%)$ with turbulators containing round holes with diameter $d=5 \mathrm{~mm}$ can reach $1.59,1.55,1.52$ at most compared with D-I water in circular tube when $l=5 \mathrm{~cm}, l=10 \mathrm{~cm}, l=15 \mathrm{~cm}$.

\subsubsection{Exergy efficiency evaluation}

The thermal efficiency evaluation can only evaluate the economic efficiency of the cooling system. Thus, there is a method to explore exergy efficiency to reflect the quality of energy. Fig. 31 reflects the exergy efficiency of enhanced tube with turbulators based on equation (16). Through theoretical analysis, it is easy to find that the entire coordinate system is divided into four regions (I, II, III, IV) by three boundary lines. Area I indicates that the working conditions in this area can intensify the heat transfer when the mass flow rate is identical. Area II indicates that the conditions in this area can intensify the heat transfer when the pumping power is identical, while they can weaken the heat transfer when the mass flow rate is the same. Area III indicates that when the differential pressure is identical, the working conditions in this area can intensify heat transfer, but those begin to be weakened when the pumping power is the same. Area IV indicates that the working conditions in this area can weaken the heat transfer as the differential pressure is identical. In Fig. 31, all points of this experimental system are in Area I and II, which means that when 

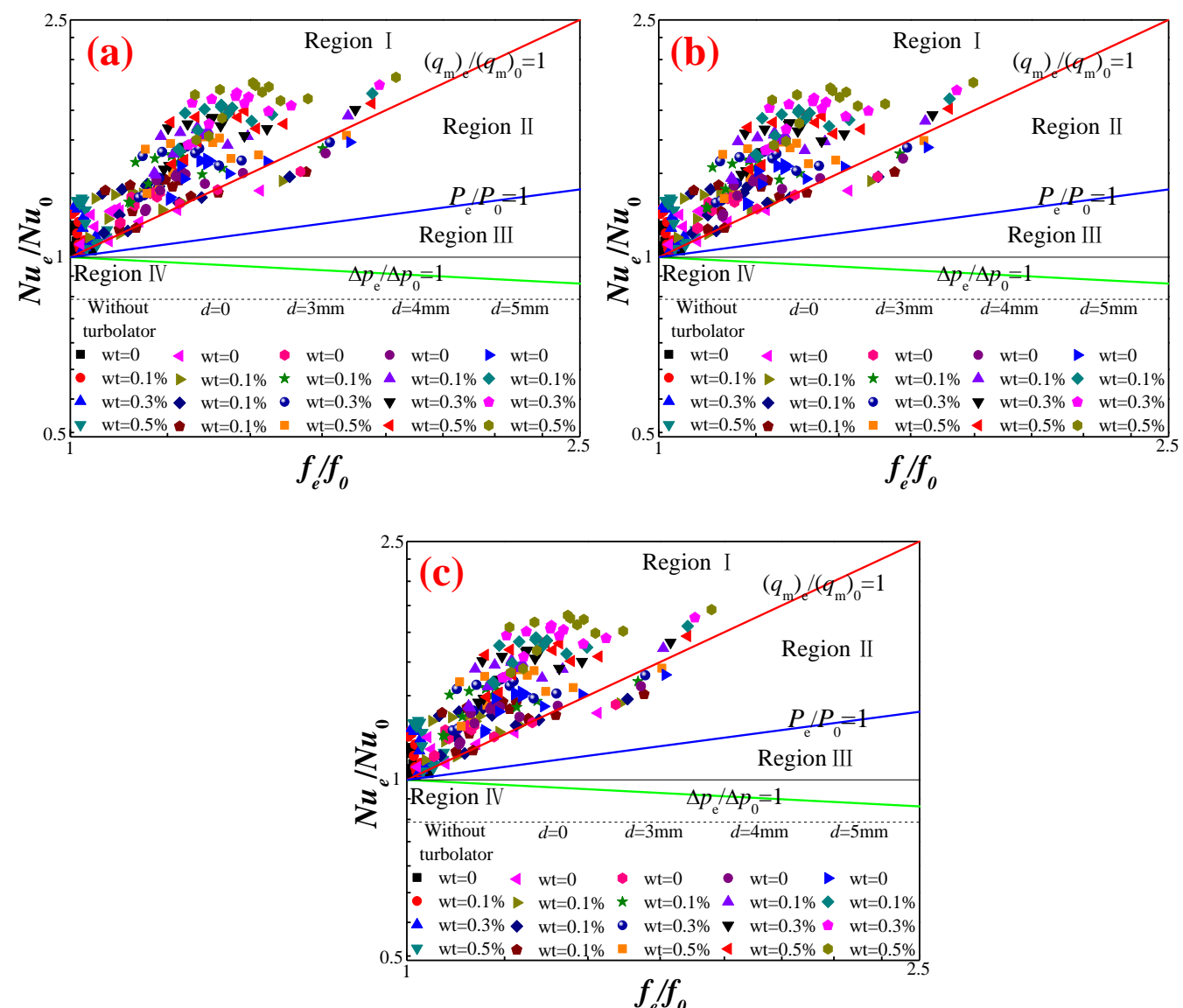

Fig. 31. Effects of round hole diameter on exergy efficiency evaluation index, (a) $l=5 \mathrm{~cm}$, (b) $l=10 \mathrm{~mm}$, (c) $l=15 \mathrm{~mm}$

In the exergy efficiency region, all working points above-mentioned are

721 exhibited. Results indicate that all the working conditions are advantageous to

722 enhance the heat transfer performance. Fig. 32 shows the slope of each point

723 above-mentioned in a histogram. It is shown that for three different conditions, the exergy efficiency increases with mass fraction, and nanofluids have the highest exergy

725 efficiency when $\omega=0.5 \%$, which means that nanoparticles are advantageous to 726 enhance exergy efficiency. Also, the maximum of it can be got when the Re reaches 6000. 

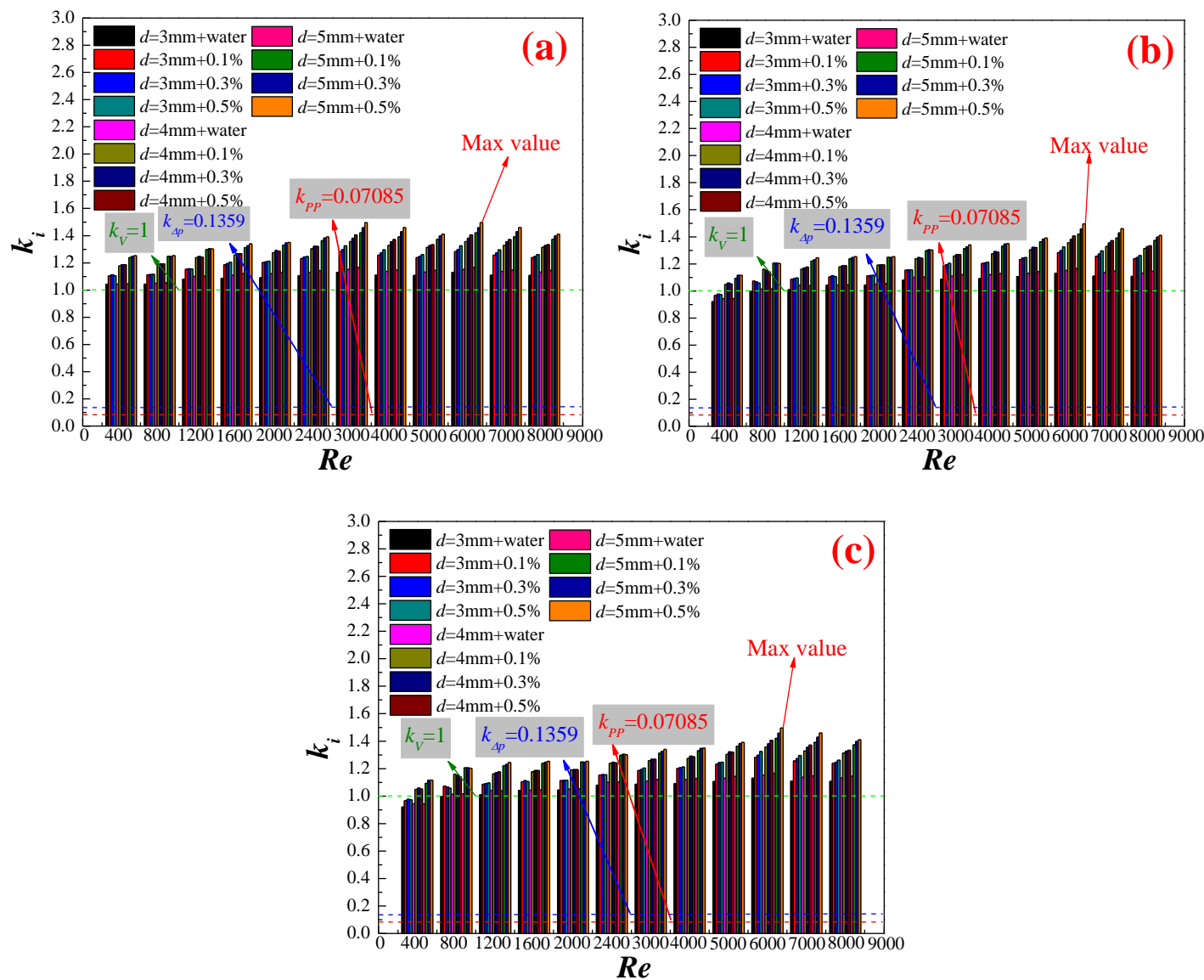

Fig. 32. The slopes of results in Fig. 31 , (a) $l=5 \mathrm{~cm}$, (b) $l=10 \mathrm{~mm}$, (c) $l=15 \mathrm{~mm}$

\section{Conclusions}

In this paper, the effects of turbulators containing various diameter and pitch-row

round holes on the thermal and hydraulic characteristics of nanofluids in a triangular

tube are explored. At the same time, the following conclusions can be obtained:

(1) $N u$ numbers always augments with nanoparticle mass fractions. High fraction

can enhance the heat transfer by $16.73 \%$ most.

(2) Triangular tube with turbulator containing round holes with diameter $d=5 \mathrm{~mm}$

shows the largest heat transfer enhancement ratio. It can improve the $N u$ by $6.498 \%$, diameter $d=3 \mathrm{~mm}$ respectively. 
742

743

enhancement ratio. Triangular tube with turbulator containing round holes with pitch-row $l=5 \mathrm{~cm}$ can improve the $N u$ by $8.947 \%, 10.002 \%, 10.504 \%$ at best when $d=3 \mathrm{~mm}, d=4 \mathrm{~mm}, d=5 \mathrm{~mm}$ compared with round hole pitch-row $l=15 \mathrm{~mm}$ respectively.

(4) Thermal efficiency (comprehensive evaluation index) augments with $R e$ at the beginning, and subsequently declines with $R e$. The highest thermal efficiency occurs near $R e=6000$, which is mentioned as the critical $R e$ above.

(5) Thermal efficiency augments with the increasing round hole diameter and the decreasing round hole pitch-row. The index in the triangular tube can reach 1.59 at most.

(6) Most working conditions show excellent exergy efficiency when the mass flow rate is uniform. The max value can be reached when $R e=6000$ and $\omega=0.5 \%$.

\section{Acknowledgements}

This work is financially supported by "National Natural Science Foundation of China" (Grant No. 51606214), "Natural Science Foundation of Jiangsu Province, China" (Grant No. BK20181359) and "EU ThermaSMART project, H2020-MSCA-RISE (778104)-Smart thermal management of high power microprocessors using phase-change (ThermaSMART)".

\section{References}

[1] J. Zeng, Y. Xuan, Tunable full-spectrum photo-thermal conversion features of magnetic-plasmonic $\mathrm{Fe}_{3} \mathrm{O}_{4} / \mathrm{TiN}$ nanofluid, Nano Energy 51 (2018) 754-763.

[2] X. Liu, Y. Xuan, Full-spectrum volumetric solar thermal conversion via photonic nanofluids, Nanoscale 9(39) (2017) 14854-14860. 
764

765

766

767

768

769

770

771

772

773

774

775

776

777

778

779

780

781

782

783

784

[3] J. Zeng, Y. Xuan, Enhanced solar thermal conversion and thermal conduction of MWCNT-SiO 2 /Ag binary nanofluids, Appl. Energ. 212 (2018) 809-819.

[4] X. Liu, Y. Xuan, Defects-assisted solar absorption of plasmonic nanoshell-based nanofluids, Sol. Energy 146 (2017) 503-510.

[5] X. Wang, Y. He, X. Liu, G. Cheng, J. Zhu, Solar steam generation through bio-inspired interface heating of broadband-absorbing plasmonic membranes, Appl. Energ. 195 (2017) 414-425.

[6] X. Liu, X. Wang, J. Huang, G. Cheng, Y. He, Volumetric solar steam generation enhanced by reduced graphene oxide nanofluid, Appl. Energ. 220 (2018) 302-312.

[7] J.Q. Li, L.W. Mou, J.Y. Zhang, Y.H. Zhang, L.W. Fan, Enhanced pool boiling heat transfer during quenching of water on superhydrophilic porous surfaces: Effects of the surface wickability, Int. J. Heat Mass Transf. 125 (2018) 494-505.

[8] J.Q. Li, L.W. Mou, Y.H. Zhang, Z.S. Yang, M.H. Hou, L.W. Fan, Z.T. Yu, An experimental study of the accelerated quenching rate and enhanced pool boiling heat transfer on rodlets with a superhydrophilic surface in subcooled water, Exp. Therm. Fluid Sci. 92 (2018) 103-112.

[9] N. Zhao, L. Guo, C. Qi, T. Chen, X. Cui, Experimental study on thermo-hydraulic performance of nanofluids in CPU heat sink with rectangular grooves and cylindrical bugles based on exergy efficiency, Energy Convers. Manage. 181 (2019) 235-246. 
[10]C. Qi, N. Zhao, X. Cui, T. Chen, J. Hu, Effects of half spherical bulges on heat transfer characteristics of CPU cooled by $\mathrm{TiO}_{2}$-water nanofluids, Int. J. Heat Mass Transf. 123 (2018) 320-330.

[11]N. Zhao, C. Qi, T. Chen, J. Tang, X. Cui, Experimental study on influences of cylindrical grooves on thermal efficiency, exergy efficiency and entropy generation of CPU cooled by nanofluids, Int. J. Heat Mass Transf. 135 (2019) $16-32$.

[12]L. Shi, Y. He, Y. Hu, X. Wang, Thermophysical properties of $\mathrm{Fe}_{3} \mathrm{O}_{4} @ \mathrm{CNT}$ nanofluid and controllable heat transfer performance under magnetic field, Energy Convers. Manage. 177 (2018) 249-257.

[13]Y. Guo, D. Qin, S. Shen, R. Bennacer, Nanofluid multi-phase convective heat transfer in closed domain: simulation with lattice Boltzmann method, Int. Commun. Heat Mass Transf. 39(3) (2012) 350-354.

[14]A.H. Pordanjani, A. Jahanbakhshi, A.A. Nadooshan, M. Afrand, Effect of two isothermal obstacles on the natural convection of nanofluid in the presence of magnetic field inside an enclosure with sinusoidal wall temperature distribution, Int. J. Heat Mass Transf. 121 (2018) 565-578.

[15]M. Nojoomizadeh, A. Karimipour, M. Firouzi, M. Afrand, Investigation of permeability and porosity effects on the slip velocity and convection heat transfer rate of $\mathrm{Fe}_{3} \mathrm{O}_{4} /$ water nanofluid flow in a microchannel while its lower half filled by a porous medium, Int. J. Heat Mass Transf. 119 (2018) 891-906. 
[16]H. Teimouri, G.A. Sheikhzadeh, M. Afrand, M.M. Fakhari, Mixed convection in a rotating eccentric annulus containing nanofluid using bi-orthogonal grid types: a finite volume simulation, J. Mol. Liq. 227 (2017) 114-126.

[17]M.A. Sheremet, I. Pop, O. Mahian, Natural convection in an inclined cavity with time-periodic temperature boundary conditions using nanofluids: Application in solar collectors, Int. J. Heat Mass Transf. 116 (2018) 751-761.

[18]M.A. Sheremet, D.S. Cimpean, I. Pop, Free convection in a partially heated wavy porous cavity filled with a nanofluid under the effects of Brownian diffusion and thermophoresis, Appl. Therm. Eng. 113 (2017) 413-418.

[19]I.V. Miroshnichenko, M.A. Sheremet, H.F. Oztop, N. Abu-Hamdeh, Natural convection of $\mathrm{Al}_{2} \mathrm{O}_{3} / \mathrm{H}_{2} \mathrm{O}$ nanofluid in an open inclined cavity with a heat-generating element, Int. J. Heat Mass Transf. 126 (2018) 184-191.

[20]I.V. Miroshnichenko, M.A. Sheremet, H.F. Oztop, N. Abu-Hamdeh, Natural convection of alumina-water nanofluid in an open cavity having multiple porous layers, Int. J. Heat Mass Transf. 125 (2018) 648-657.

[21]O. Pourmehran, M. Rahimi-Gorji, D.D. Ganji, Heat transfer and flow analysis of nanofluid flow induced by a stretching sheet in the presence of an external magnetic field, J. Taiwan Inst. Chem. E. 65 (2016) 162-171.

[22]M. Rahimi-Gorji, O. Pourmehran, M. Gorji-Bandpy, D.D. Ganji, Unsteady squeezing nanofluid simulation and investigation of its effect on important heat transfer parameters in presence of magnetic field, J. Taiwan Inst. Chem. E. 67 (2016) 467-475. 
[23]O. Pourmehran, M.M. Sarafraz, M. Rahimi-Gorji, D.D. Ganji, Rheological behaviour of various metal-based nano-fluids between rotating discs: a new insight, J. Taiwan Inst. Chem. E. 88 (2018) 37-48.

[24]O. Pourmehran, M. Rahimi-Gorji, M. Hatami, S.A.R. Sahebi, G. Domairry, Numerical optimization of microchannel heat sink (MCHS) performance cooled by KKL based nanofluids in saturated porous medium, J. Taiwan Inst. Chem. E. 55 (2015) 49-68.

[25]M. Izadi, R. Mohebbi, D. Karimi, M.A. Sheremet, Numerical simulation of natural convection heat transfer inside a $\perp$ shaped cavity filled by a MWCNT-Fe ${ }_{3} \mathrm{O}_{4} /$ water hybrid nanofluids using LBM, Chem. Eng. Process. 125 (2018) 56-66.

[26]M. Izadi, G. Hoghoughi, R. Mohebbi, M. Sheremet, Nanoparticle migration and natural convection heat transfer of $\mathrm{Cu}$-water nanofluid inside a porous undulant-wall enclosure using LTNE and two-phase model, J. Mol. Liq. 261 (2018) 357-372.

[27]M. Izadi, R. Mohebbi, A.A. Delouei, H. Sajjadi, Natural convection of a magnetizable hybrid nanofluid inside a porous enclosure subjected to two variable magnetic fields, Int. J. Mech. Sci. 151 (2019) 154-169.

[28]M. Izadi, S. Sinaei, S.A.M. Mehryan, H.F. Oztop, N. Abu-Hamdeh, Natural convection of a nanofluid between two eccentric cylinders saturated by porous material: Buongiorno's two phase model, Int. J. Heat Mass Transf. 127 (2018) $67-75$. 
850

851

852

853

854

855

856

857

858

859

860

861

862

863

864

865

866

867

868

869

870

871

[29] O. Mahian, A. Kianifar, S.Z. Heris, S. Wongwises, Natural convection of silica nanofluids in square and triangular enclosures: theoretical and experimental study, Int. J. Heat Mass Transf. 99 (2016) 792-804.

[30]E. Shahsavani, M. Afrand, R. Kalbasi, Using experimental data to estimate the heat transfer and pressure drop of non-Newtonian nanofluid flow through a circular tube: applicable for use in heat exchangers, Appl. Therm. Eng. 129 (2018) 1573-1581.

[31]M. Sheikholeslami, Magnetohydrodynamic nanofluid forced convection in a porous lid driven cubic cavity using Lattice Boltzmann method, J. Mol. Liq. 231 (2017) 555-565.

[32]M. Sheikholeslami, T. Hayat, A. Alsaedi, Numerical simulation of nanofluid forced convection heat transfer improvement in existence of magnetic field using lattice Boltzmann method, Int. J. Heat Mass Transf. 108 (2017) 1870-1883.

[33]M. Sheikholeslami, A.J. Chamkha, Influence of Lorentz forces on nanofluid forced convection considering Marangoni convection, J. Mol. Liq. 225 (2017) 750-757.

[34]M. Sheikholeslami, M.M. Bhatti, Forced convection of nanofluid in presence of constant magnetic field considering shape effects of nanoparticles, Int. J. Heat Mass Transf. 111 (2017) 1039-1049.

[35]M. Sheikholeslami, T. Hayat, A. Alsaedi, S. Abelman, Numerical analysis of EHD nanofluid force convective heat transfer considering electric field 
dependent viscosity, Int. J. Heat Mass Transf. 108 (2017) 2558-2565.

[36]M. Sheikholeslami, M. Seyednezhad, Simulation of nanofluid flow and natural convection in a porous media under the influence of electric field using CVFEM, Int. J. Heat Mass Transf. 120 (2018) 772-781.

[37]M. Sheikholeslami, M. Jafaryar, M. Hedayat, A. Shafee, Z. Li, T.K. Nguyen, M. Bakouri, Heat transfer and turbulent simulation of nanomaterial due to compound turbulator including irreversibility analysis, Int. J. Heat Mass Transf. 137 (2019) 1290-1300.

[38]M. Sheikholeslami, Influence of magnetic field on $\mathrm{Al}_{2} \mathrm{O}_{3}-\mathrm{H}_{2} \mathrm{O}$ nanofluid forced convection heat transfer in a porous lid driven cavity with hot sphere obstacle by means of LBM, J. Mol. Liq. 263 (2018) 472-488.

[39]P. Naphon, S. Wiriyasart, Pulsating flow and magnetic field effects on the convective heat transfer of $\mathrm{TiO}_{2}$-water nanofluids in helically corrugated tube, Int. J. Heat Mass Transf. 125 (2018) 1054-1060.

[40]P. Naphon, S. Wiriyasart, T. Arisariyawong, T. Nualboonrueng, Magnetic field effect on the nanofluids convective heat transfer and pressure drop in the spirally coiled tubes, Int. J. Heat Mass Transf. 110 (2017) 739-745.

[41]P. Naphon, S. Wiriyasart, Pulsating $\mathrm{TiO}_{2} /$ water nanofluids flow and heat transfer in the spirally coiled tubes with different magnetic field directions, Int. J. Heat Mass Transf. 115 (2017) 537-543.

[42]P. Naphon, S. Wiriyasart, T. Arisariyawong, Artificial neural network analysis the pulsating Nusselt number and friction factor of $\mathrm{TiO}_{2} /$ water nanofluids in the 
spirally coiled tube with magnetic field, Int. J. Heat Mass Transf. 118 (2018) 1152-1159.

[43]X. Zhou, X. Li, K. Cheng, X. Huai, Numerical study of heat transfer enhancement of nano liquid-metal fluid forced convection in circular tube, ASME J. Heat Transf. 140(8) (2018) 081901.

[44]B. Sun, A. Yang, D. Yang, Experimental study on the heat transfer and flow characteristics of nanofluids in the built-in twisted belt external thread tubes, Int. J. Heat Mass Transf. 107 (2017) 712-722.

[45]D. Yang, B. Sun, H. Li, C. Zhang, Y. Liu, Comparative study on the heat transfer characteristics of nano-refrigerants inside a smooth tube and internal thread tube, Int. J. Heat Mass Transf. 113 (2017) 538-543.

[46]C. Qi, M. Liu, T. Luo, Y. Pan, Z. Rao, Effects of twisted tape structures on thermo-hydraulic performances of nanofluids in a triangular tube, Int. J. Heat Mass Transf. 127(Part C) (2018) 146-159.

[47]C. Qi, M. Liu, J. Tang, Influence of triangle tube structure with twisted tape on the thermo-hydraulic performance of nanofluids in heat-exchange system based on thermal and exergy efficiency, Energy Convers. Manage. 192 (2019) 243-268.

[48]S. Mei, C. Qi, T. Luo, X. Zhai, Y. Yan, Effects of magnetic field on thermo-hydraulic performance of $\mathrm{Fe}_{3} \mathrm{O}_{4}$-water nanofluids in a corrugated tube, Int. J. Heat Mass Transf. 128 (2019) 24-45.

[49]S. Mei, C. Qi, M. Liu, F. Fan, L. Liang, Effects of paralleled magnetic field on 
thermo-hydraulic performances of $\mathrm{Fe}_{3} \mathrm{O}_{4}$-water nanofluids in a circular tube, Int. J. Heat Mass Transf. 134 (2019) 707-721.

[50]C. Qi, G. Wang, Y. Yan, S. Mei, T. Luo, Effect of rotating twisted tape on thermo-hydraulic performances of nanofluids in heat-exchanger systems, Energy Convers. Manage. 166 (2018) 744-757.

[51]C. Qi, L. Yang, T. Chen, Z. Rao, Experimental study on thermo-hydraulic 
inserts. Int. J. Heat Mass Transf. 106 (2017) 877-883.

939 [57]Y.L. Wan, Study on natural convection and forced convection heat transfer characteristics of $\mathrm{TiO}_{2}$-water nanofluids, Master thesis, China University of

[58]J. Maxwell, A treatise on electricity and magnetism, Vol. 1, Clarendon Press, Oxford, 1873.

[59]Brinkman H. The viscosity of concentrated suspensions and solutions, J. Chem. Phys. 20(4) (1952): 571-571.

[60]B.C. Pak, Y.I. Cho, Hydrodynamic and heat transfer study of dispersed fluids 2012. (in Chinese) 
961 [66]E. Smithberg, F. Landis, Friction and forced convection heat-transfer 962 characteristics in tubes with twisted tape swirl generators, J. Heat Transf. 86(1) $963 \quad$ (1964) 39-48.

964 [67]C. Qi, L. Liang, Z. Rao, Study on the flow and heat transfer of liquid metal base 965 nanofluid with different nanoparticle radiuses based on two-phase lattice Boltzmann method, Int. J. Heat Mass Transf. 94 (2016) 316-326.

967 [68]C. Qi, G. Wang, L. Yang, Y. Wan, Z. Rao, Two-phase lattice Boltzmann 968 simulation of the effects of base fluid and nanoparticle size on natural 969 convection heat transfer of nanofluid, Int. J. Heat Mass Transf. 105 (2017) $664-672$. 